\title{
Abundance of gap junctions at glutamatergic mixed synapses in adult Mosquitofish spinal cord neurons
}

\author{
Jose L. Serrano-Velez' , Melanie Rodriguez-Alvarado ${ }^{1}$, Irma I. Torres-Vazquez ${ }^{1}$, Scott E. Fraser ${ }^{2}$, \\ Thomas Yasumura ${ }^{3}$, Kimberly G. Vanderpool ${ }^{3}$, John E. Rash ${ }^{3,4}$ and Eduardo Rosa-Molinar,5* \\ ' Biological Imaging Group, University of Puerto Rico, San Juan, PR, USA \\ ${ }^{2}$ Molecular and Computational Biology Section, University of Southern California, Los Angeles, CA, USA \\ ${ }^{3}$ Department of Biomedical Sciences, Colorado State University, Fort Collins, CO, USA \\ ${ }^{4}$ Program in Molecular, Cellular and Integrative Neurosciences, Colorado State University, Fort Collins, CO, USA \\ ${ }^{5}$ Institute of Neurobiology, School of Medicine, University of Puerto Rico, San Juan, PR, USA
}

\section{Edited by:}

Benjamin R. Arenkiel, Baylor

College of Medicine, USA

Reviewed by:

Audrey Mercer, University College

London, UK

Brian Burrell, University of South

Dakota, USA

\section{*Correspondence:}

Eduardo Rosa-Molinar, Biological Imaging Group, University of Puerto Rico, P.O. Box 21809 UPR Station, San Juan, PR 00931-1809, USA e-mail: ed@hpcf.upr.edu

\begin{abstract}
"Dye-coupling", whole-mount immunohistochemistry for gap junction channel protein connexin 35 (Cx35), and freeze-fracture replica immunogold labeling (FRIL) reveal an abundance of electrical synapses/gap junctions at glutamatergic mixed synapses in the 14th spinal segment that innervates the adult male gonopodium of Western Mosquitofish, Gambusia affinis (Mosquitofish). To study gap junctions' role in fast motor behavior, we used a minimally-invasive neural-tract-tracing technique to introduce gap junction-permeant or -impermeant dyes into deep muscles controlling the gonopodium of the adult male Mosquitofish, a teleost fish that rapidly transfers (complete in $<20 \mathrm{mS}$ ) spermatozeugmata into the female reproductive tract. Dye-coupling in the 14th spinal segment controlling the gonopodium reveals coupling between motor neurons and a commissural primary ascending interneuron (CoPA IN) and shows that the 14th segment has an extensive and elaborate dendritic arbor and more gap junctions than do other segments. Whole-mount immunohistochemistry for $\mathrm{C} \times 35$ results confirm dye-coupling and show it occurs via gap junctions. Finally, FRIL shows that gap junctions are at mixed synapses and reveals that $>50$ of the 62 gap junctions at mixed synapses are in the 14th spinal segment. Our results support and extend studies showing gap junctions at mixed synapses in spinal cord segments involved in control of genital reflexes in rodents, and they suggest a link between mixed synapses and fast motor behavior. The findings provide a basis for studies of specific roles of spinal neurons in the generation/regulation of sex-specific behavior and for studies of gap junctions' role in regulating fast motor behavior. Finally, the CoPA IN provides a novel candidate neuron for future studies of gap junctions and neural control of fast motor behaviors.
\end{abstract}

Keywords: connexin 35/36, connexins, dye-coupling, freeze-fracture replica immunogold labeling, gap junctions, mixed synapses, neurons, spinal cord

\section{INTRODUCTION}

Electrical synapses, hereafter, gap junctions, are abundant, are evidenced by widespread coupling at birth, and until the central nervous system (CNS) matures, play a role in shaping and patterning neuronal connectivity in the invertebrate ${ }^{[1]}$ and vertebrate ${ }^{[2]}$ CNS ( ${ }^{[1]}$ Furshpan and Potter, 1959; Bennett et al., 1963; Robertson

\footnotetext{
Abbreviations: AFB-594, Alexa Fluor ${ }^{\circledR}-594$ Biocytin; Mini-Emerald, Dextran, Fluorescein and Biotin, 10,000 MW, Anionic, Lysine Fixable; MNs, motor neurons; CoPA IN, commissural primary ascending interneuron; CNS, central nervous system; Cx35/36, connexin 35/connexin 36; FRIL, freezefracture replica immunogold labeling; GFAP, glial fibrillary acid protein; IMP, intramembrane particle; LE, labeling efficiency; $\mathrm{sCM}$, spinning-disk confocal microscopy; $\mathrm{LMC}^{\text {lateral, }}$, lateral lateral motor column; $\mathrm{LMC}^{\text {medial }}$, medial lateral motor column; MLF, medial longitudinal fasciculus; MMC, medial motor column; DV, dorso-ventral; PBS, phosphate buffered saline; PBSS, phosphate buffered saline with saponin; RT, room temperature.
}

et al., 1963; Furshpan, 1964; Sotelo and Korn, 1978; Fulton et al., 1980; Vaney, 1991; Walton and Navarrete, 1991; Dermietzel and Spray, 1993; Peinado et al., 1993a,b; Kalb, 1994; Kandler and Katz, 1995; Wolszon, 1995; Laird, 1996; Rash et al., 1996; Bennett, 1997, 2000; Dermietzel, 1998; Edwards et al., 1999; Personius et al., 2001; Cohen-Cory, 2002; Herberholz et al., 2002; Mentis et al., 2002; Lewis and Eisen, 2003; Pereda et al., 2003; Scheiffele, 2003; Bennett and Zukin, 2004; Connors and Long, 2004; Montoro and Yuste, 2004; Szabo et al., 2004; Fan et al., 2005; MarinBurgin et al., 2005, 2006, 2008; Phelan, 2005; Waites et al., 2005, ${ }^{[2]}$ Arumugam et al., 2005; Kamasawa et al., 2006; Chen et al., 2007; Chuang et al., 2007; McAllister, 2007; Norman and Maricq, 2007; Szabo and Zoran, 2007; Todd et al., 2010; Whelan, 2010; Hoge et al., 2011; Park et al., 2011; Hamzei-Sichani et al., 2012; Lynn et al., 2012; Sugimoto et al., 2013; Bautista and Nagy, 2014). As the CNS matures, gap junction uncoupling occurs as the initial 
electrical synapses switch to chemical synapses and the former concomitantly decrease in number in the adult.

Axo-axonal or dendrodendritic gap junctions are probably best known for coupling parvalbumin-containing $\gamma$-aminobutyric acid (GABA)ergic interneurons (Ins)to facilitate the synchronization of rhythmic oscillatory activity for neuronal communication in the adult neocortex (Galarreta and Hestrin, 1999, 2001a,b; Gibson et al., 1999; Fukuda and Kosaka, 2000, 2003; Bartos et al., 2002; Muller et al., 2005, 2006). Gap junction coupling has been identified in numerous neural microcircuits and has been linked to motor behavior; however, the extent to which gap junctions are linked to fast motor behavior has not been fully explored (Saint-Amant and Drapeau, 2000, 2001; Tresch and Kiehn, 2000; Bonnot et al., 2002; Drapeau et al., 2002; Kiehn and Tresch, 2002; Hervé et al., 2004; Rash et al., 2004, 2013; Söhl et al., 2005; Goodenough and Paul, 2009; Vervaeke et al., 2010; Pereda et al., 2013).

To assist in understanding gap junctions' role in fast motor behavior, we used a minimally-invasive neural-tracttracing/labeling technique to introduce either gap junctionpermeant or -impermeant dyes into deep muscles controlling the gonopodium (a sexually dimorphic sperm transferring organ) of a "reference species", the adult male Western Mosquitofish, Gambusia affinis (Mosquitofish) a small, sexually dimorphic teleost fish whose radical remodeling and shifting of the axial and appendicular musculoskeletal support facilitates an extremely rapid movement of the gonopodium to transfer encapsulated sperm bundles, spermatozeugmata, into the adult female reproductive tract (Rosa-Molinar et al., 1994, 1996, 1998; Rosa-Molinar, 2005; Rivera-Rivera et al., 2010).

To transfer spermatozeugmata, the male Mosquitofish body bends into an "S-shaped fast-start" curvature defined as "torque" (Figure 1); simultaneously the gonopodium makes an extremely rapid directional movement defined as "thrust" (Figure 1; Weihs, 1973; Webb, 1976; Harper and Blake, 1990, 1991; Johnston et al., 1995; Rosa-Molinar et al., 1996; Domenici and Blake, 1997; Spierts and Leeuwen, 1999; Hale, 2002; Rosa-Molinar, 2005; Rivera-Rivera et al., 2010). The speed of the "torque/thrust" maneuver (complete in $<20 \mathrm{mS}$ ), particularly of the "thrust" component, suggests that electrical and not chemical synapses are involved in controlling the finer aspects of Mosquitofish rapid motor behavior.

A simple dye-coupling assay combined with spinning disk confocal microscopy shows spinal motor neurons are dye-coupled to

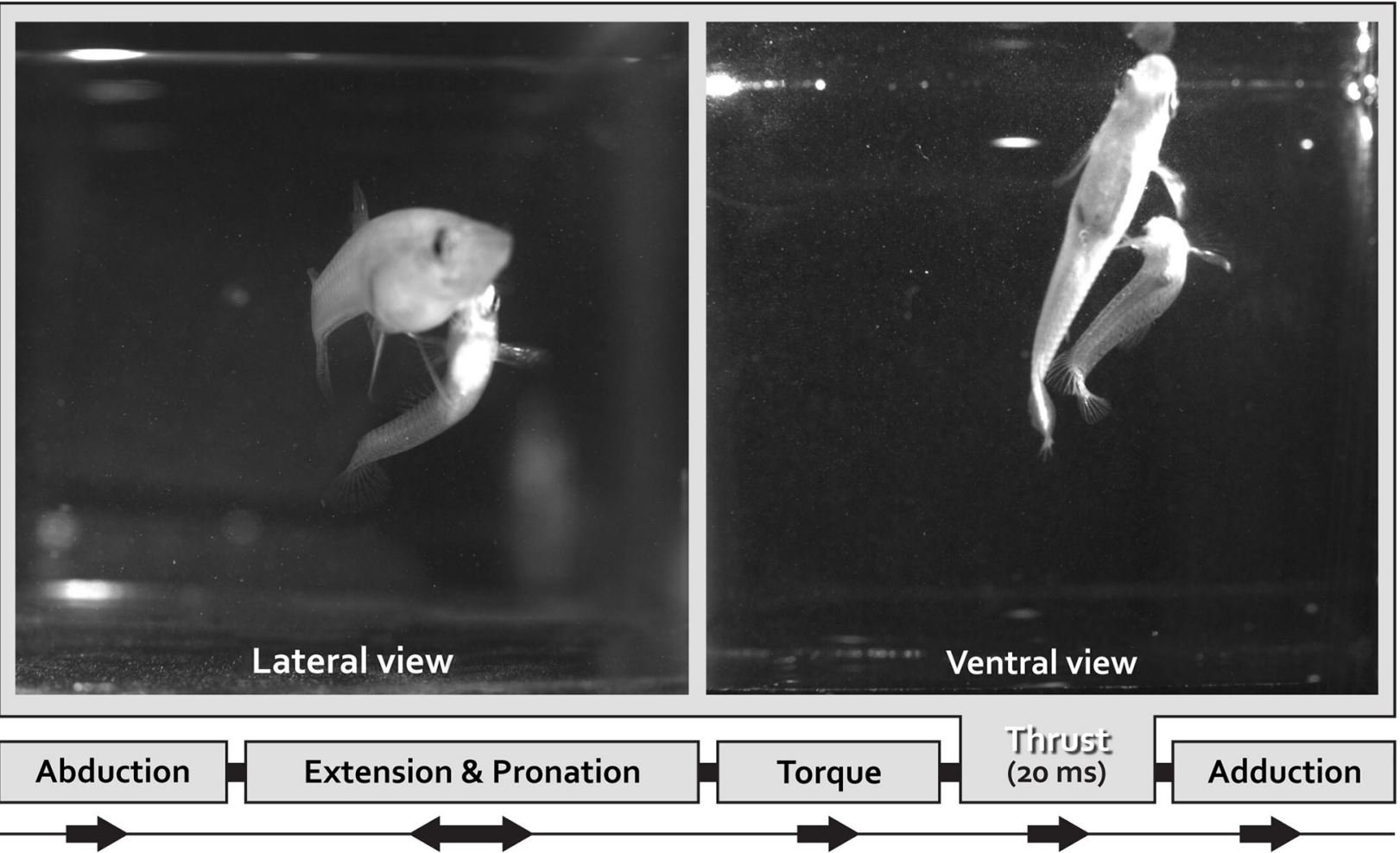

FIGURE 1 | One lateral and one ventral view of coital behavioral sequences filmed using high speed video at 500 oframeses-1.frames for a male Mosquitofish show the rapid movement portion of the circumduction of the gonopodium. With the gonopodium abducted, the male approaches the female from behind and directly underneath her and then adducts the gonopodium. Just prior to circumducting, the gonopodium is extended and pronated to a point that is nearly parallel with the body. To transfer spermatozeugmata, the male Mosquitofish bends his body into an S-shape-type fast-start-like movement (see lateral and ventral view) during the torque-thrust motion of the circumduction of the gonopodium. The sequence ends with the gonopodium adducted. We analyzed the speed of the circumduction of the gonopodium (the specific movements were: abduction, extension and pronation, torque, thrust, and adduction), specifically the fastest portion of the sequence, the "thrust" movement $(20 \mathrm{~ms})$. 
interneurons and reveals their unique arborization patterns and morphologies. Whole-mount immunohistochemistry combined with spinning disk confocal microscopy shows the dye-coupling to be via $\mathrm{Cx} 35 / 36$ puncta (i.e., gap junctions). Freeze-facture replica immunogold labeling (FRIL) confirms the immunohistochemistry results and reveals that the Cx35/36 puncta are, in fact, gap junctions at mixed synapses. Our results demonstrate the occurrence and abundance of axo-dendritic gap junctions at glutamatergic synapses between dye-coupled spinal motor neurons and interneurons in the adult Mosquitofish, particularly in a spinal region controlling an innate fast coital behavior of the adult male. The fundamental data and insights reported in this paper provide a basis for on-going work to unambiguously determine the connexin composition in apposed axo-dendritic gap junctions' hemiplaques at glutamatergic synapses and to differentially map connexin distribution within the arbors of dye-coupled spinal motor neurons and interneurons in the adult Mosquitofish. The results also move us closer to attaining a clear understanding of the fundamental role of gap junctions in sculpting complex arborization patterns, morphologies, and synaptic connectivity of neurons during development and maturation of the CNS.

\section{MATERIALS AND METHODS}

Eighty wild-type adult (female $n=40$; male $n=40$ ) Western Mosquitofish, Gambusia affinis (hereafter Mosquitofish) were used. All experimental procedures and care were approved and conducted according to Principles of Laboratory Animal Care (NIH publication No. 86-23, Rev. 1985 (Rosario-Ortiz et al., 2008)) and the University of Puerto Rico-Rio Piedras Institutional Animal Care and Use Committee guidelines. All fish were collected and maintained under permits issued by the Puerto Rico Department of Natural Resources.

\section{DYE-COUPLING ASSAY}

The 80 adult Mosquitofish were anesthetized by immersion in pasteurized tank water plus dilute benzocaine (1:2000). Filter paper fibers saturated with a gap junction-permeant dye (0.32 kDa Alexa Flour ${ }^{\circledR}-594$ Biocytin; hereafter AFB-594) or with a mixture of a gap junction-permeant dye, $0.32 \mathrm{kDa}$ AFB-594 and a gap junction-nonpermeant dye $(10 \mathrm{kDa}$ Dextran, Fluorescein and Biotin, Anionic, Lysine Fixable; hereafter Mini-Emerald) were surgically implanted directly into nerves innervating the deep muscles (musculus erector analis major, musculus erector analis, and musculus depressor analis) of the adult male gonopodium, the sexually dimorphic genitalia, and into the deep muscles of the adult female anal fin; Mosquitofish were revived and the dye was allowed to transport $6.0 \mathrm{~h}$, which is sufficient to obtain Golgi-like filling of spinal motor neurons (MNs) and INs. Mosquitofish were euthanized by immersion in pasteurized tank water containing benzocaine (1:4000) and intracardially perfused with teleost buffer $\mathrm{pH} 7.4$, followed with $2.0 \%$ formaldehyde made from an $8 \%$ aqueous depolymerized paraformaldehyde (PFA) diluted in teleost buffer $\mathrm{pH}$ 7.4. The spinal cord associated with vertebral segments $7-17$ was removed, dissected-free, and post-fixed overnight with 2\% PFA in teleost buffer $\mathrm{pH}$ 7.4. Spinal cords were covered with mounting medium (Vectashield ${ }^{\circledR}$ Vector Laboratories, Burlingame, CA) and cover-slipped. Spinal cord whole-mount preparations were viewed and digitally photographed using a Nikon CFI Super Fluor 20X objective (N.A. 0.50; W.D. 2.10) and a Nikon CFI Plan Fluor 60X 0.11-0.23 correction collar spring load objective (N.A. 0.85; W.D. 0.30) in a Nikon Eclipse 800 epi-fluorescence microscope with the appropriate single pass epi-fluorescence filters and a fluorescence illumination system $\left(\mathrm{XCite}^{\mathrm{TM}} 120\right)$ to attain optimal fluorescence detection efficiency. High-resolution images taken using a Qimaging Retiga Exi 12-bit CCD camera with a HRF50L1 High Resolution $0.5 \mathrm{x}$ coupler captured large areas and neurocytological details of MNs and interneurons (INs), specifically, commissural primary ascending interneurons (CoPA INs). The Nikon E800's peripheral components were controlled by NIS Elements Advance Research software. Spinal cord whole-mount preparations were also viewed with a Nikon C1 Laser Scanning Confocal Microscope (LSCM) using a Nikon CFI Super Fluor 20X objective (N.A. 0.50; W.D. 2.10) and a Nikon CFI-PLAN APO 60X objective with correction collar and spring load (N.A. 0.85; W.D. 0.30). The light path consisted of $543 \mathrm{~nm}$ excitation, with collection using a long pass $560 \mathrm{~nm}$ filter or a $633 \mathrm{~nm}$ excitation, with collection using a long pass $650 \mathrm{~nm}$ filter. A PerkinElmer UltraView $^{\text {TM }}$ Spinning Disk Confocal scan head mounted on a Zeiss Axiovert Microscope was also used to view spinal cord wholemount preparations.

A simple dye-coupling assay was used to validate the gap junctional dye-coupling reported in this paper. In 100\% of adult male and female Mosquitofish, retrograde labeling using either a low-molecular gap junction-permeant dye, $0.32 \mathrm{kDa}$ AFB-594 (red fluorescence channel only; Figure 2A), a high-molecular weight gap junction-nonpermeant dye, $10 \mathrm{kDa}$ Mini-Emerald (green fluorescence channel only; Figure 2B), or a mixture of a low-molecular gap junction-permeant dye, $0.32 \mathrm{kDa}$ AFB594 (red and green fluorescence channel overlay; Figure 2C), and a high-molecular weight gap junction-nonpermeant dye, $10 \mathrm{kDa}$ Mini-Emerald (red and green fluorescence channel overlay; Figure 2C), revealed "dye-coupling" (red fluorescence in coupled spinal neurons; Figure 2C), defined as the movement of a gap junction-permeant dye (i.e., $0.32 \mathrm{kDa}$ AFB-594 [red, Figure 2C]) from spinal neuron to spinal neuron.

It is important to note that the overlay channel seen in Figure 2C differs from the co-localization channel seen in Figure 2D. The overlay channel seen in Figure 2C shows all of the pixel information within a selected region of interest (ROI), including pixel information that appears in the same spatial location within the retrogradely-filled spinal neurons. The colocalization channel seen in Figure 2D shows only pixel information that appears in the same spatial location within the retrogradely-filled spinal neurons that can be seen in both the green and red fluorescence channels as seen in Figures 2B,C, respectively. We set the lower threshold limit to 900 and set the upper threshold limit based on the highest intensity level of pixels within the soma of the MN's in a ROI. Note that this threshold was applied equally to the red and green fluorescence channels for all of the images used in our co-localization analysis. Note, too, that the excitation and emission spectra (Figure 2E) were carefully selected to avoid cross-talk and bleed-through of the gap junction-permeant and gap junction-non-permeant dyes. 

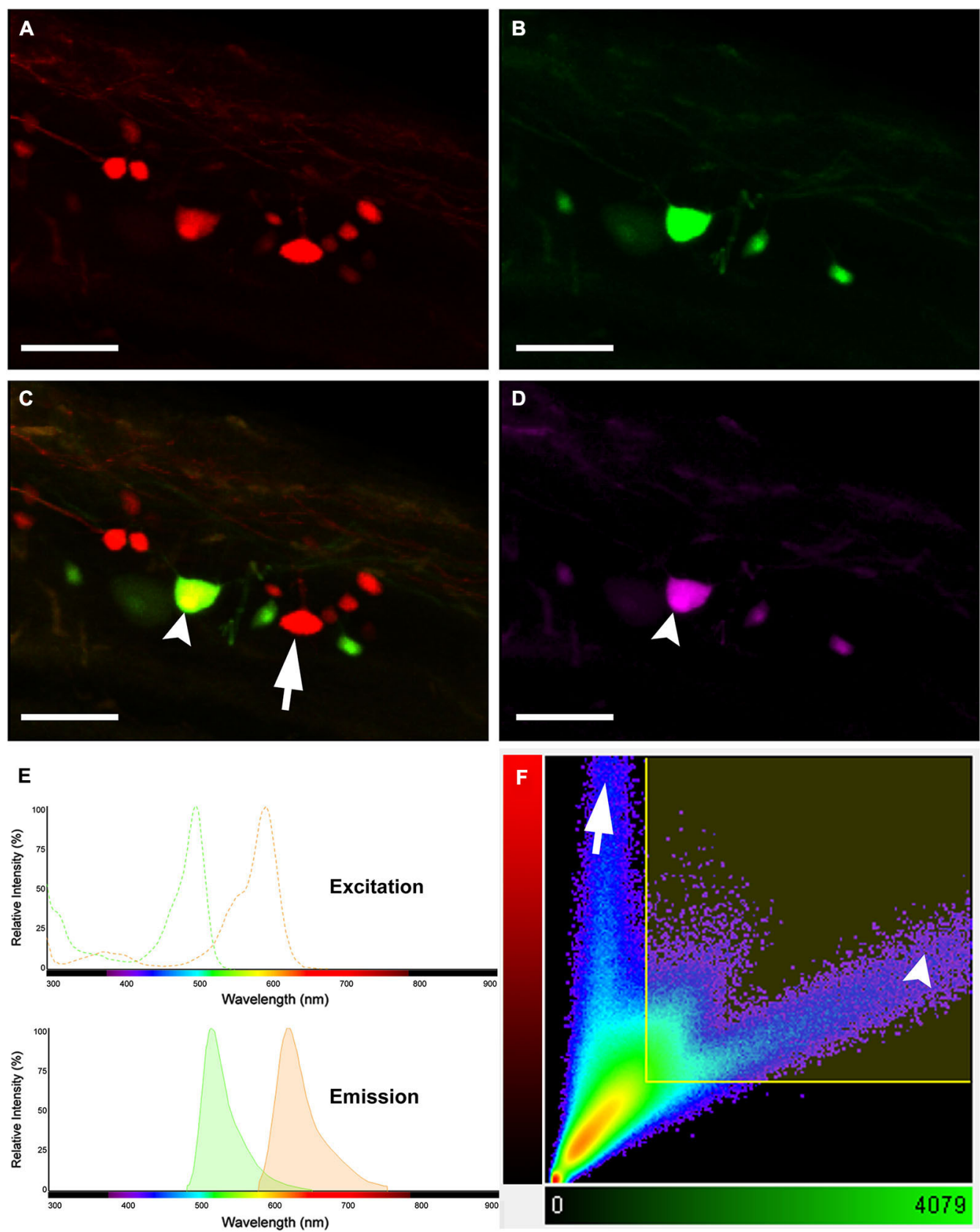

FIGURE 2 | Images showing double labeling AFB-594 (red) and Mini-Emerald (green) revealing dye-coupling between spinal motor neurons. AFB-594 (A) and Mini-Emerald (B) retrogradely labeling revealed motor neurons from the 14th ventral root. We merge red and green channels (C) to demonstrate that Mini-Emerald retrograde labeling was restrict to fewer cells, presumably to motor neurons projecting to the periphery. A three-dimensional threshold co-localization analysis confirmed, as seen in the co-localization channel (D), that AFB-594 retrogradely labeling diffuse to neighbor cells (arrow) in contrast to Mini-Emerald (arrow head). (E) Excitation and Emission spectra were carefully selected to avoid cross-talk and bleed-through of the dyes. Finally, $\mathbf{F}$ shows the co-localization scatter plot, the selected region was tresholded based on the average intensity of the somas (900) on each channel. Pearson's coefficient in the co-localized volume for this analysis was 0.5172 . Calibration bar equals $50 \mu \mathrm{m}$.
The scatterplot (Figure $2 \mathrm{~F}$ ) reveals the intensity contribution of both the red fluorescence channel (vertical axis) and the green fluorescence channel (horizontal axis) in the pixel values of the analyzed images. Note that pure signal from each single channel tends to be close to the corresponding axis. In Figure $\mathbf{2 C}$, the dye-coupled spinal neuron contained "pure signal" from the
AFB-594 (red fluorescence channel) and is represented in the scatterplot by the pixels outside the threshold box and close to the vertical axis (see Figure 2F). In contrast, since the spinal neurons labeled with Mini-Emerald also are labeled with AFB-594, the scatterplot reveals the co-localized pixels corresponding to those spinal neurons distributed far from the horizontal axis (green 
fluorescence channel), thus, confirming the positive correlation between the two dyes (see Figure 2F). We used the Pearson's correlation coefficient as a measure of the correlation of the intensity distribution between each channel. Pearson's correlation coefficient in the co-localized volume for this analysis was 0.5172 .

\section{WESTERN BLOTS}

Western blots (WB) of tissue extracts from vertebrae 8-16 of intact adult Mosquitofish spinal cords, brains, and livers were used to detect female/male differences in gap junction protein expression. The spinal cords, brains, and livers of adult females $(N=28$; groups $n=4)$ and males $(N=28$, groups $n=4$ ) were dissected out. Sample tissues were immediately frozen in dry ice and homogenized or were kept at $-80^{\circ} \mathrm{C}$ until use. WB protocol was performed as previously described, with few minor modifications (Vega et al., 2005, 2008). Tissues were pooled, weighed, and homogenized in radioimmunoprecipitation (RIPA) buffer (1X) [Cell Signaling Technology, Beverly, MA, USA] containing 1\% protease inhibitor cocktail (PIC; Sigma Aldrich, St. Louis, MO) and $1 \mathrm{mM}$ phenylmethylsulfonyl fluoride (PMSF; Sigma Aldrich, St. Louis, MO). Insoluble materials were removed by centrifugation at $14,000 \mathrm{rpm}$ for $5 \mathrm{~min}$ at $4^{\circ} \mathrm{C}$. The Bradford assay (BioRad, Hercules, CA, USA) was used to estimate the approximate protein concentration of the supernatants by detecting change in absorbance using a spectrophotometer (DU730 Beckman Coulter, Brea, CA, USA). Then, samples were diluted in loading buffer $(60 \mathrm{mM}$ Tris- $\mathrm{HCl}[\mathrm{pH} 6.8], 5 \%$ 2-metacaptoethanol, 2\% sodium dodecyl sulfate (SDS), 10\% glycerol, 0.025\% Bromophenol Blue) in the appropriate volume to load $40 \mu \mathrm{g}$ of protein per well. The supernatants, loading control, and the molecular weight marker (Precision Plus Protein Standards, Bio-Rad, Hercules, CA, USA) were electrophoresed on SDS-polyacrylamide gel (SDSPAGE 12\%). For immunoblotting, the separated proteins were transferred onto pure nitrocellulose membrane $(0.45 \mu \mathrm{m}$, BioRad, Hercules, CA, USA) for $1 \mathrm{hr}$ at $4^{\circ} \mathrm{C}$ at a constant voltage of $100 \mathrm{~V}$. Membranes were then stained with Ponceau S to corroborate transfer of proteins; then they were blocked with 5\% skim milk in tris (hydroxymethyl) aminomethane [Tris] Buffered Saline with Tween ${ }^{\circledR} 20$ (polyoxyethylene sorbitane monolaureate) [TBST] Buffer (1xTBS: 25 mM Tris Base, $150 \mathrm{mM} \mathrm{NaCl}, 30 \mathrm{mM} \mathrm{KCl}$; and $0.1 \%$ Tween-20; hereafter, blocking buffer) for $1 \mathrm{hr}$. Following the blocking step, membranes were incubated overnight at $4^{\circ} \mathrm{C}$ with either a mouse anti-Connexin (Cx)35/36 monoclonal antibody; clone: 9D7.2 (1:250 [Cat. \# MAB3043, Chemicon]; EMD Millipore, Billerica, MA, USA) or a mouse anti-Cx35/36 monoclonal antibody, clone 8F6.2 (1:250; Cat. \# MAB3045, Chemicon; EMD Millipore, Billerica, MA, USA) diluted in blocking buffer. Membranes were washed several times to remove unbound Cx35/36 antibody and incubated with Goat anti-Mouse Poly-horseradish peroxidase (HRP; 1:2000; Cat. \# 2230, Thermo Fisher Scientific, Suwanee, GA, USA) for $1 \mathrm{hr}$ at room temperature (RT). The recognized immunoreactive bands were detected using enhanced chemiluminescence reactions according to manufacturer's instructions (SuperSignal West Femto Maximum Sensitive Substrate [Cat.\#
34095]; or SuperSignal West Dura Extended Duration Substrate [Cat. \# 34075] Thermo Fisher Scientific, Suwanee, GA, USA). Then, the membranes were exposed in the ChemiDoc ${ }^{\mathrm{TM}}$ XRS+ System with Image Lab $^{\mathrm{TM}}$ software (Bio-Rad, Hercules, CA, USA). For spinal cord and liver samples, membranes were stripped and reprobed for loading with anti-glyceraldehyde 3phosphate dehydrogenase (GAPDH)-HRP conjugated (1:1000; Cat. \# ab105428, Abcam, Cambridge, MA, USA). For brain samples, monoclonal anti-acetylated tubulin (1:1000; Cat. \# T6793, Sigma Aldrich, St. Louis, MO, USA) was used. Then, membranes were visualized as described above. Optical density (OD) was expressed as a ratio of Cx35/36 and GAPDH or acetylated $\alpha$-tubulin (Loading Control proteins [LC]) by using a densitometer and the Image Lab $^{\mathrm{TM}}$ software (Bio-Rad, Hercules, CA, USA). Statistical analysis was performed with Prism 6.0 software (GraphPad Software, Inc., San Diego, CA, USA) and Office Excel 2007 (Microsoft Redmond, WA, USA). Results are reported as mean $\pm \mathrm{SEM}$; all the $p$-values were calculated by Student's $t$-test $\left({ }^{*} p<0.05\right)$. Western blots (Figures $3 \mathrm{~A}, \mathrm{C}$ ) and band density (Figures 3B,D) of the mouse anti-Cx35/36 monoclonal antibodies (MAB3045 and MAB3043) show that both antibodies detect differences in $\mathrm{Cx} 35 / 36$ expression (see Figures 3A-D). Note that only mouse anti-Cx35/36 monoclonal antibody (MAB3043) revealed significant sex differences in $\mathrm{Cx} 35 / 36$ expression in spinal cord tissue homogenates (see Figures 3C,D).

\section{CONNEXIN 35/36 WHOLE-MOUNT IMMUNOHISTOCHEMISTRY}

We used the primary anti-Cx35/36 antibody anti-Cx35 MAB3043 (Millipore, Billerica, MA). Spinal cord whole-mounts were rinsed with phosphate buffered saline $(\mathrm{PBS})[3 \times 10 \mathrm{~min}$ each at RT] to remove fixative and permeabilized in a solution of PBS and $0.1 \%$ Saponin (PBSS) for $4 \mathrm{~h}$ at RT. Spinal cord wholemounts were incubated with blocking buffer (3\% Normal Goat Serum/PBSS) for $1 \mathrm{~h}$ at RT then incubated overnight at $4^{\circ} \mathrm{C}$ with the anti-Cx35/36 MAB3043. Following several rinses at RT with PBS $[6 \times 10$ min each], the spinal cord whole-mounts were incubated with secondary antibody (Alexa Fluor ${ }^{\circledR} 488$ Goat anti-Mouse [Invitrogen, Carlsbad, CA]) for $3 \mathrm{~h}$ at RT. Unbound antibody was removed by rinsing tissues with PBS at RT $[6 \times 10 \mathrm{~min}$ each]. The spinal cord whole-mounts were incubated with blocking buffer for $30 \mathrm{~min}$ at RT and were incubated with second primary antibodies or nuclear stained and mounted, as described below. All second primary antibodies were diluted in blocking buffer and incubated overnight at $4^{\circ} \mathrm{C}$. Then, the spinal cord whole-mounts were rinsed several times with PBS to remove unbound second primary antibody, and the appropriate secondary antibody was added. As controls for the whole-mount immunohistochemical procedures, eye (positive control) and liver tissue (negative control) were incubated in blocking solution without primary or secondary antibody (i.e., NGS/PBSS only) followed by the standard protocol to validate the specificity of the antibody. Spinal cord, eye, and liver wholemounts were covered with mounting medium (Vectashield ${ }^{\circledR}$ Vector Laboratories, Burlingame, CA) and cover-slipped. Spinal cord, eye, and liver whole-mounts were then viewed and digitally photographed using a Nikon CFI Super Fluor 20X 
A

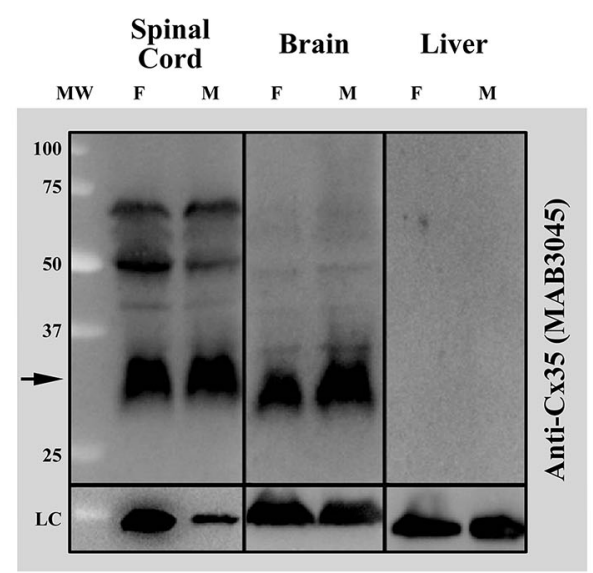

C

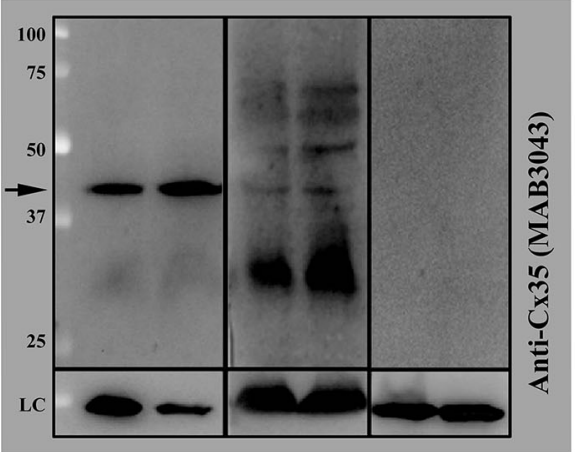

E

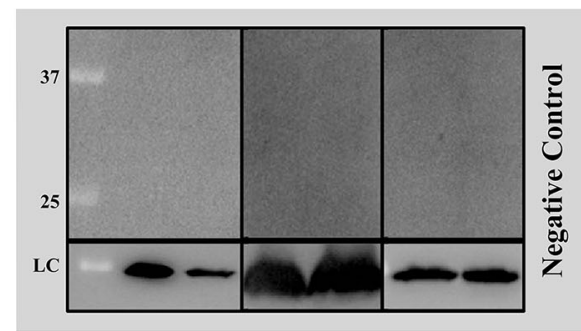

B

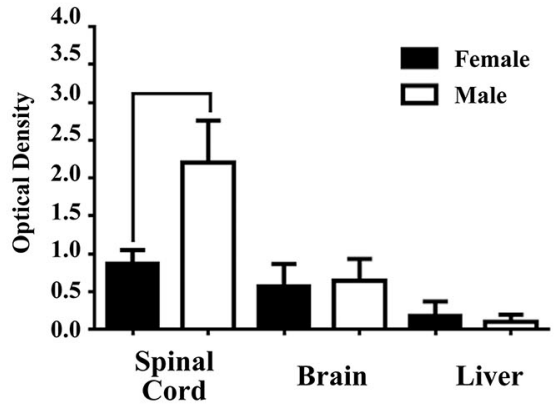

D

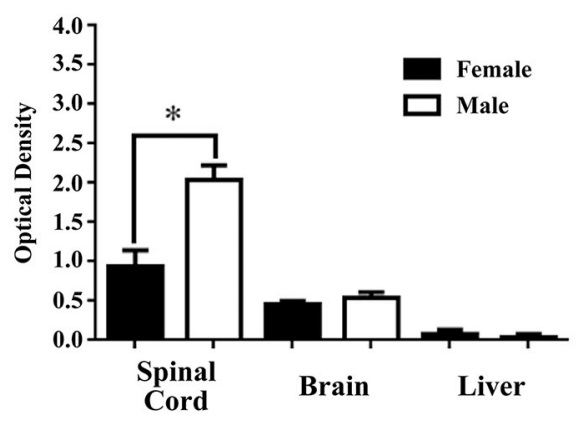

$\mathbf{F}$

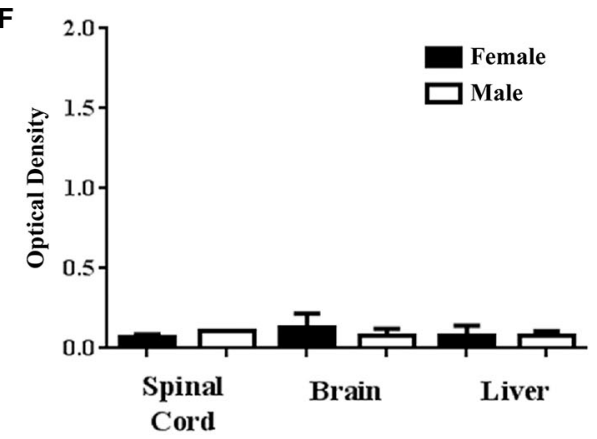

anti-mouse Poly-HRP antibody. The WB and densitometric analysis (see Figures $\mathbf{1 E}, \mathbf{F}$ ) of this negative control validates the results of the commercially available mouse anti-Cx35/36 monoclonal antibodies by demonstrating that the specific bands recognized are not associated with a non-specific binding from the secondary antibody. OD at each protein concentration was averaged (3 blots), showing differences determined by using each potential loading control (anti-GAPDH or anti-acetylated-tubulin). Statistical differences were determined with student $t$-test analysis $\left({ }^{*} p<\right.$ 0.05). Each lane contains $40 \mu \mathrm{g}$ of the extracted proteins from selected tissues homogenates of adult female and male Mosquitofish. The difference in the band intensity in the loading controls (LC) of the spinal cord tissue homogenate of male and female Mosquitofish could be interpreted as an issue in the total protein loaded in the WB (see Figures $\mathbf{1 A}, \mathbf{C}, \mathbf{E}$ ). However, the densitometric analysis (see Figures $\mathbf{1 B}, \mathbf{D}, \mathbf{F}$ ) demonstrates a difference between males and females. objective (N.A. 0.50; W.D. 2.10) and a Nikon CFI Plan Fluor 60X 0.11-0.23 correction collar spring load objective (N.A. 0.85; W.D. 0.30) in a Nikon Eclipse 800 epi-fluorescence microscope with the appropriate single pass epi-fluorescence filters and a fluorescence illumination system $\left(\mathrm{XCite}^{\mathrm{TM}} 120\right)$ to attain optimal fluorescence detection efficiency. High resolution images 

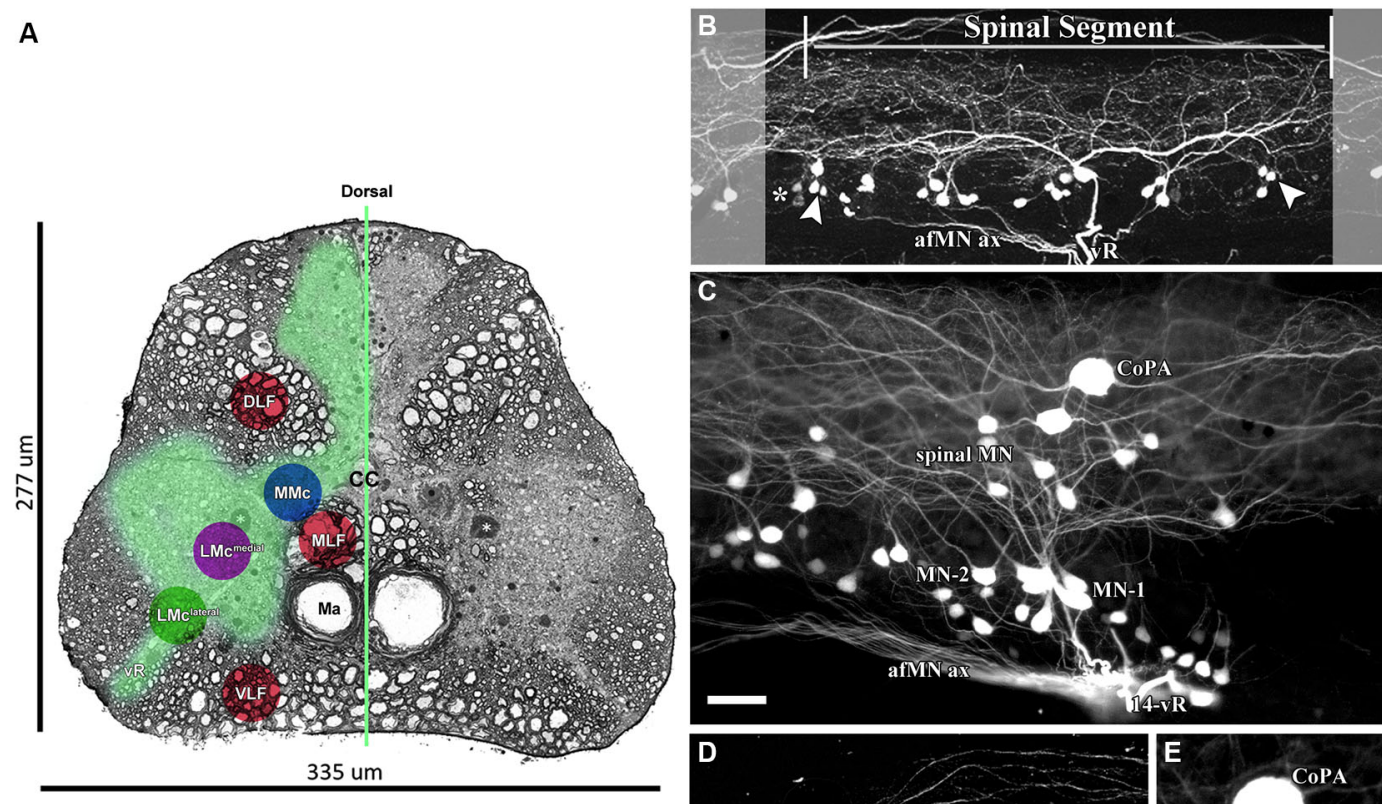

Ventral
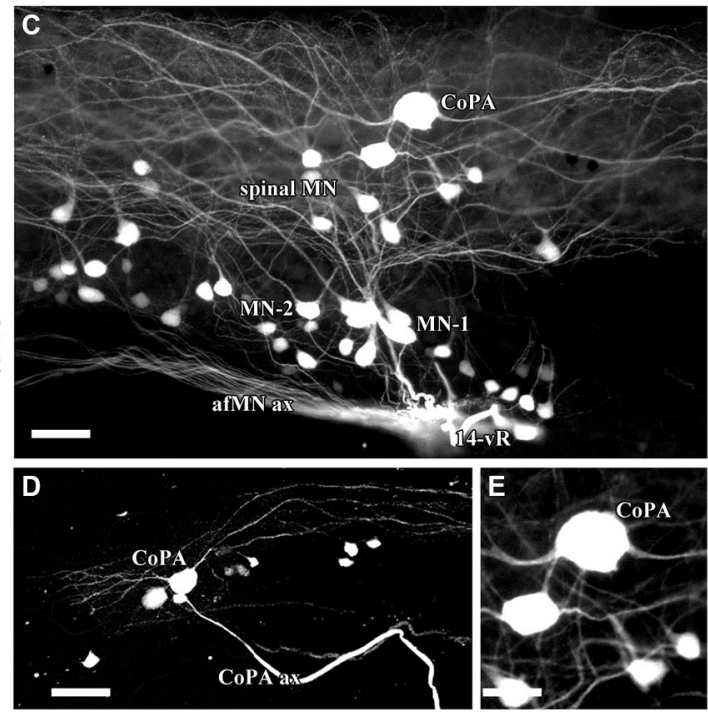

FIGURE 4 | 0.32 kDa AFB-594 revealing dye-coupling between spinal motor neurons (MN) and a CoPA interneuron (IN). (A) Serial block-face scanning electron micrograph showing a cross-sectional view of the spinal cord at the 14th ventral root. The AFB-594 motor neurons were visualized with Avidin-Biotin-Peroxidase/DAB reaction and are located in the Medial Lateral Motor Column (LMCmedial). (B) Extensive dye-coupling spanned across three (14th-16th) spinal segments. (C) 0.32 kDa AFB-594 retrograde labeling reveal presumptive dye-coupling between $\mathrm{MNs}$ and an IN of the commissural primary ascending (CoPA) class. D and $\mathbf{E}$ higher magnification views show CoPA INs are located dorsally to the MNs in the spinal cord, have a bi-polar T-shaped dendritic arbor with fine dendrites extending rostrally and caudally from the soma into the dorsal longitudinal fasciculus, and a long axon [CoPA ax] projects ventrally. Calibration bar equals (C and D) $50 \mu \mathrm{m}$ and (E) $30 \mu \mathrm{m}$. taken using a Qimaging Retiga Exi 12-bit CCD camera with a HRF50L1 High Resolution 0.5x coupler captured large areas and neurocytological details of $\mathrm{MNs}$ and interneurons INs, specifically, CoPA INs.

\section{FRIL}

The FRIL protocol has been described in detail (Rash and Yasumura, 1999; Pereda et al., 2003). The labeled spinal cord region associated with vertebral segments $7-17$ ( $n=12$ females; $n=12$ males; see labeling section above for details) was placed in a 3\% low melting agarose, then transferred and embedded in $6 \%$ agarose, followed by refrigeration until fully gelled. Coronal sections (100 $\mu \mathrm{m}$-thick) and longitudinal sections were cut using a Lancer Vibrotome 3000 (Technical Products, Inc., St. Louis, MO, USA) that maintained the tissue sections at $4^{\circ} \mathrm{C}$. Spinal cord sections were infiltrated with 30\% glycerol, mounted on aluminum planchettes, and frozen by contact with a liquid nitrogen-cooled metal mirror (i.e., Ultra-Freeze MF 7000; RMC Products, Tucson, AZ, USA). Frozen samples were fractured and replicated in a JEOL/RMC 9010 freeze-fracture device, then bonded to gold "index" grids by using 2.0\% Lexan (GE Plastics, Pittsfield, MA, USA) dissolved in dichloroethane. After solvent evaporation at $-25^{\circ} \mathrm{C}$, the Lexan-stabilized samples were thawed, viewed, and digitally photographed using a 5X $(0.15$ N.A. Fluar) or 10X (0.5 N.A.; Plan Neofluar) objective in a Zeiss 510 Meta Laser Scanning Confocal Microscope (Carl Zeiss MicroImaging, Thornwood, NY, USA). Replicas were washed in $2.5 \%$ SDS detergent in $0.16 \%$ Tris- $\mathrm{HCl}$ buffer $(\mathrm{pH} 8.9$ ) for 29 $\mathrm{h}$ at $48.5^{\circ} \mathrm{C}$. After the initial wash in $2.5 \%$ SDS $(4.0 \mathrm{~h})$, the samples were digested $1.25 \mathrm{~h}$ in $4.0 \%$ collagenase D in $0.15 \mathrm{M}$ Sorensen's phosphate buffer ( $\mathrm{pH}$ 7.4), followed by an additional 18-24 h in SDS solution. The replicas were rinsed in "labelingblocking buffer" (1mg/mL LBB), then incubated for 1-1.5 hrs at $22-24^{\circ} \mathrm{C}$ in $1: 100$ dilution of anti-Cx35/36 antibody in LBB, which consists of $1.5 \%$ fish gelatin plus $10 \%$ heat-inactivated goat serum in Sorensen's phosphate buffer (Dinchuk et al., 1987). The antibodies used for FRIL were polyclonal anti-Cx36 (36-4600) or monoclonal anti-Cx36 (39-4200 or 37-4600) from Invitrogen, polyclonal anti-Cx35 P-Ser276 from John O'Brien (Li et al., 2009), monoclonal anti-Glutamate Receptor NMDAR1 (556308, BD Biosciences), monoclonal anti-Glutamate Receptor 2 (GluR2, MAB397, Milllipore), and three anti-pannexin 1 antibodies (no specific labeling detected; therefore antibodies not separately listed). The replicas were labeled for 12-16 hrs with speciesspecific secondary antibodies (goat anti-rabbit or goat antimouse) coupled to $6-\mathrm{nm}, 12-\mathrm{nm}$, or $18-\mathrm{nm}$ gold nanoparticles 


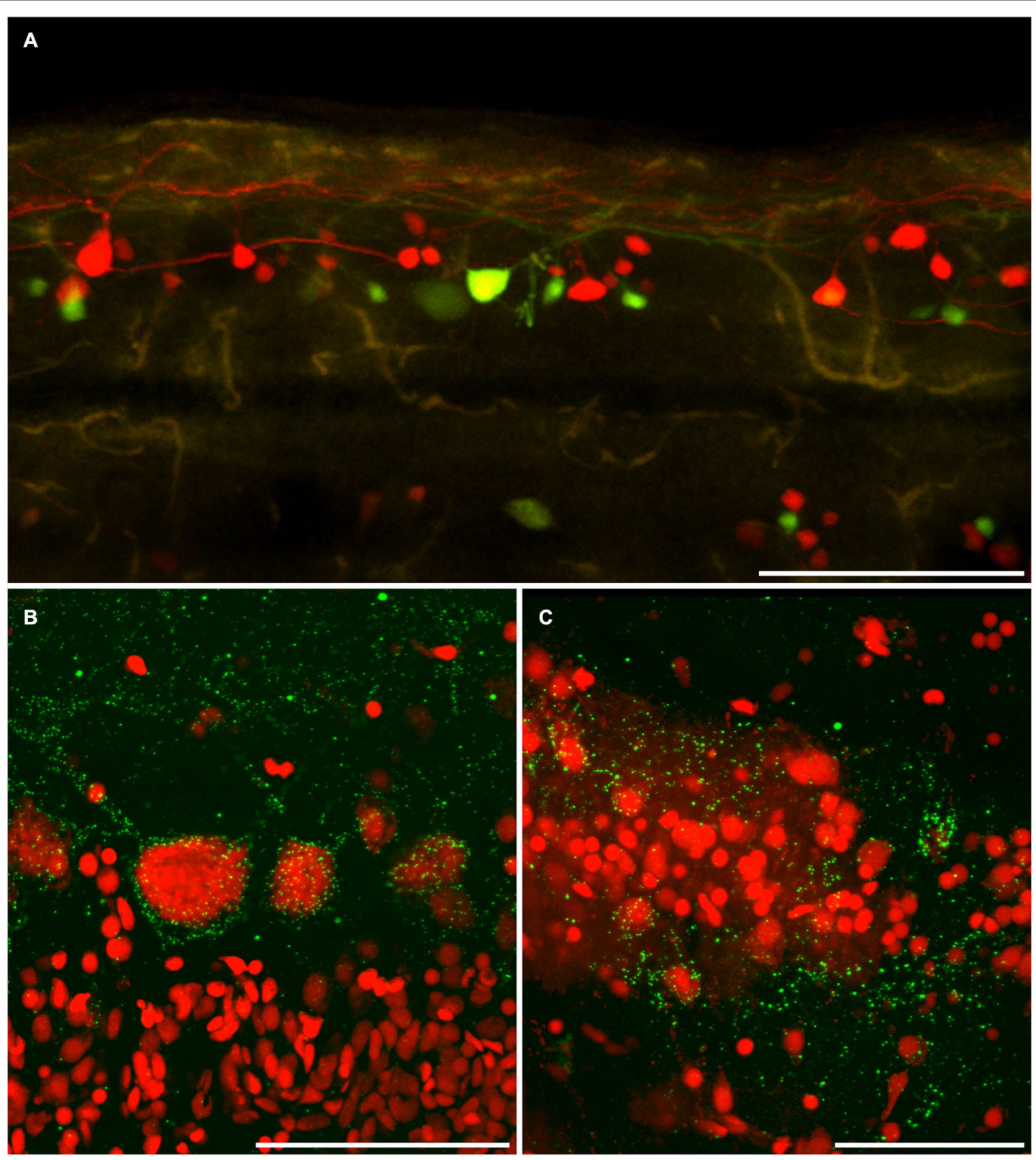

FIGURE 5 | Dye coupling is confirmed by anti-connexin $35 / 36$ immuno-labeling. (A) AFB-594 and Mini-Emerald retrograde labeling revealed motor neurons from the 14th ventral root, and showed the extensive labeling of presumably coupled neurons. Whole-mount immunohistochemistry with anti-Cx35 antibody confirms the widespread presence of connexins (green puncta) in both male (B) and female (C) Mosquitofish. Calibration bars equals (A) 200 um, (B,C) 50 um
(Jackson ImmunoResearch, Westgrove, PA, USA), or 30-nm gold nanoparticles (BBI). All FRIL replicas were viewed with a JEOL 2000 EX-II transmission electron microscope operated at 100 $\mathrm{kV}$. Stereoscopic images ( $8^{\circ}$ included angle) allowed assessment of the "sidedness" of the gold nanoparticles and the level of background immunogold labeling. Cell-specific ultrastructural markers were used to confirm cell identifications (Matsumoto et al., 1989; Rash et al., 1997), including GFAP filaments in astrocytes, spherical synaptic vesicles in axon terminals, and $10-\mathrm{nm}$
E-face IMPs in glutamatergic "asymmetric" synapses $v s$. pleomorphic synaptic vesicles and 9-nm P-face IMPs in GABAergic "symmetric" synapses (Harris and Landis, 1986; DeFelipe et al., 1988; Peters et al., 1991), and labeling for NMDA and AMPA receptors in glutamatergic mixed synapses (Rash et al., 2005; also see DeFelipe et al., 1988). FRIL images were correlated with confocal microscopic images that had been obtained before SDS washing to determine specific locations of $\mathrm{Cx} 35 / 36$ gap junction puncta. 

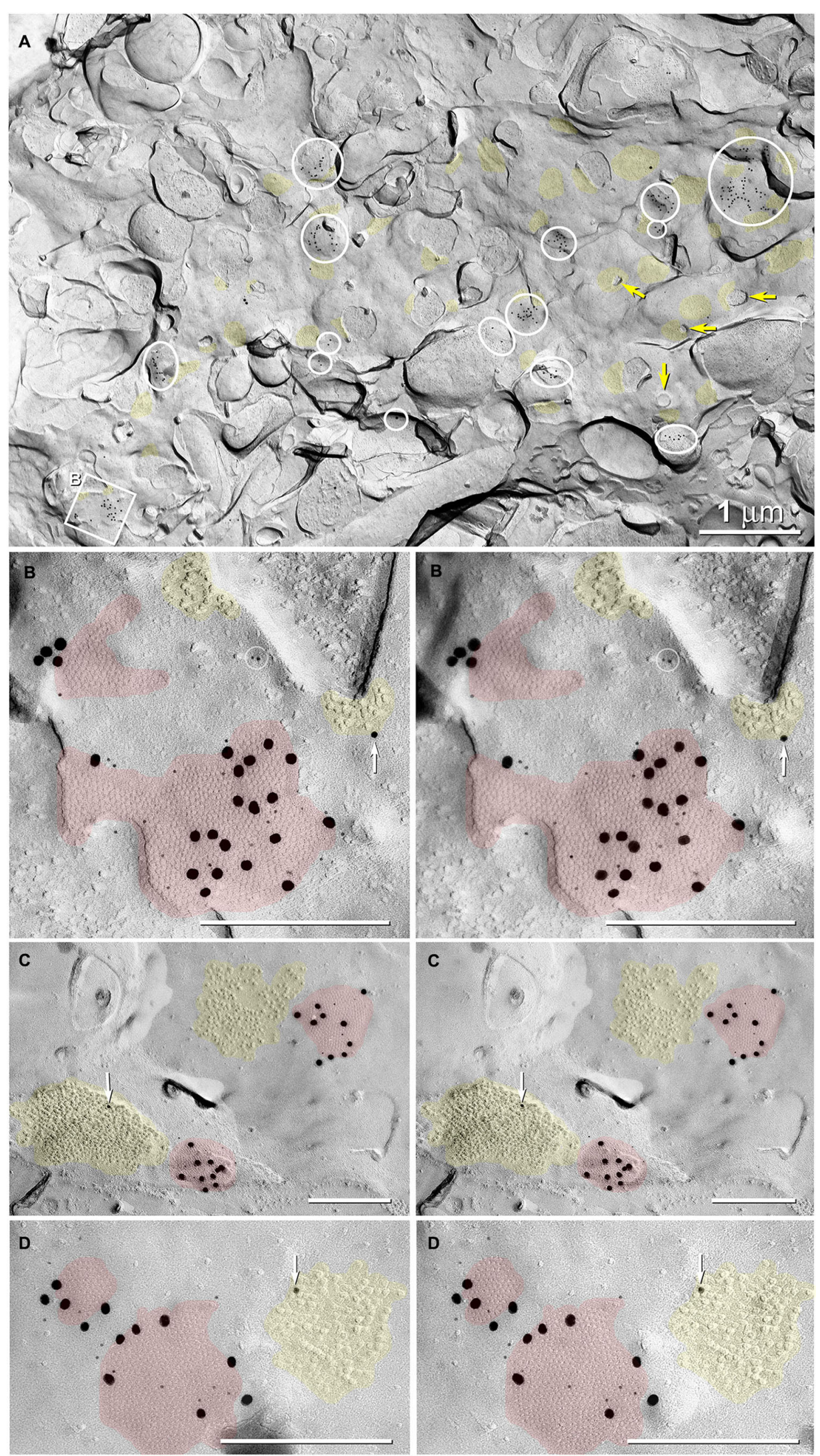

FIGURE 6 | (Continued) 


\section{FIGURE 6 | Continued}

\section{Abundance of glutamatergic mixed synapses in adult male}

Mosquitofish shown at low magnification, with selected synaptic contacts shown in higher magnification stereoscopic images. (A) The E-face of a dendrite having few spines (yellow arrows = cross-fractured necks of dendritic spines) is labeled extensively for $\mathrm{Cx35}$ by $6-\mathrm{nm}$ and

18-nm gold beads. All gap junctions are at axo-dendritic synapses (inscribed circles and inscribed square $B$ mark 20 gap junctions in this field of view); distinctive clusters of 10-nm IMPs are weakly labeled for NMDAR1 (12- and 30-nm gold nanoparticles, yellow overlays; 46 PSDs in this field of view). Some cross-fractured axon terminals contain spherical synaptic vesicles. (B) High-magnification stereoscopic view of the inscribed square " $B$ " in (A) shows two E-face gap junctions (red overlays) labeled for Cx35 (6-nm and 18-nm gold beads) and two postsynaptic clusters of E-face IMPs identified as glutamate receptors (yellow overlays) based on weak but positive labeling for NMDAR1 (12-nm gold bead, white arrow). Two 6-nm gold beads on top of the replica are circled to identify them as non-specific "noise". (C) High-magnification stereoscopic view of two E-face gap junctions (red overlays) labeled for Cx35 (6-nm and 18-nm gold beads). Each gap junction has a postsynaptic cluster of IMPs (yellow overlay) immediately adjacent to it (i.e., within $50 \mathrm{~nm}$ ). One IMP cluster is labeled for NMDAR1 (12-nm gold bead, white arrow). (D) High-magnification stereoscopic view of two E-face gap junctions (red overlays) labeled for $\mathrm{C} \times 35$ (6-nm and 18-nm gold beads): one postsynaptic cluster of IMPs (yellow overlays) is labeled for NMDAR1 (12-nm gold bead, white arrow). Unless otherwise indicated, calibration bars in all FRIL images are $0.25 \mu \mathrm{m}$, which corresponds to the limit of resolution of light microscopy in blue and green wavelengths.

\section{RESULTS}

AFB-594 retrograde labeling reveals extensive dye-coupling between MNs located in the lateral motor column (LMC) and INs located in the medial longitudinal fasciculus (MLF) of the spinal cord (Figures 4A,B). Note that this extensive dye-coupling spans three (14th-16th) spinal segments (Figure 4B), but only one spinal segment, the 14th, is shown in Figure 4B. Each spinal segment contains 44 neurons.

The 14th spinal segment, a region controlling adult male fast coital behavior, shows a more extensive and elaborate dendritic arbor (Figure 4C) than do other spinal segments. Of the 44 neurons seen in the 14th spinal segment of 12 male Mosquitofish, 28 neurons are $\mathrm{MN}-1,7$ are $\mathrm{MN}-2$, and 9 are spinal MN. Of the 44 neurons seen in the 14 th spinal segment in 12 female Mosquitofish, 29 were MN-1, 9 were MN-2, and 6 were spinal MN. The two phenotypes of motor neurons (MN-1 and MN-2) were identified based on the presence of basal and apical dendrites (see Figure 4C). The spinal neurons seen in Figure 4C are located within the medial motor column (MMC; Figure 4A) and the medial lateral motor column (LMC $^{\text {medial }}$; Figure $4 \mathrm{~A}$ ). These neurons are being characterized and identified for a subsequent report. The number of MNs was assessed by counting all of the entering axons through each of the spinal segment ventral roots and correlating them with the total number of retrogradely-filled MNs. Each of the spinal segments was confirmed by the presence of "boundary neurons" (Figure 4B).

It is clear from these results that the rapid, minimally-invasive method is selective because the only spinal neurons that send axonal processes to muscles are MNs; thus, the neuronal somas and their axonal and dendritic processes extensively filled with AFB-594 are clearly distinguishable as MNs. Dye-coupling also reveals a spinal interneuron that we identify as a commissural primary ascending interneuron (CoPA IN) based on the dorsoventral (DV) position of its soma within the MLF, and on its large slightly elongated spherical shape (see Figure $4 \mathrm{~A}$, and Figures 4C-E).

In keeping with the results of Hale et al. (2001), our results show that two distinct dendrites emerge from the rostral and caudal poles of the soma (Figure 4C) and run longitudinally across three spinal segments within the dorsal longitudinal fasciculus. Two dendrites (Figure 4E), together with the ventrally emerging axon, give the CoPA IN its characteristic T-shape (see Figures 4CE). Note that dye-coupling revealed no more than one CoPA IN per spinal segment.

The AFB-594 dye-coupling between MNs and INs in adult female and adult male Mosquitofish as seen in Figure 5A was confirmed by performing Cx35/36 whole-mount fluorescence immunohistochemistry. Figure 5B shows the high density of Cx35/36 immuno-positive gap junction puncta covering the soma and outlining the basal, apical, and proximal dendrites of MNs in male Mosquitofish; in female Mosquitofish (see Figure 5C), the density of $\mathrm{Cx} 35 / 36$ immuno-positive gap junction puncta, especially large puncta, seems greater than it does in males.

To confirm that dye-coupling between MNs and CoPA INs was mediated by Cx35-containing-gap junctions at mixed synapses, we employed FRIL and four Cx35/36 antibodies. P-Ser276 abundantly labeled pre-synaptic hemiplaques within the neuronal gap junctions (Figures 6, 7B), as was previously reported in giant club endings on Mauthner cells (Rash et al., 2013). In 6 replicas (3 males; 3 females), FRIL reveals $>115$ gap junctions are immunogold labeled for Cx35/36; 97 gap junctions are found in males and 18 in females. The immunogold labeling efficiency [LE; defined as the number of gold beads $v s$. the number of connexons counted in each gap junction (Rash and Yasumura, 1999)] ranged from 1:5 to 1:50, comparable to those of previous gap junction FRIL studies (Fujimoto, 1995; Rash et al., 2001; Pereda et al., 2003).

In 5 replicas ( 2 in adult males and 2 in adult females, each containing 6-9 spinal cord cross sections; plus 1 replica in male containing 2 longitudinal sections), we identified 35 Cx35/36 immuno-positive gap junctions in 30 appositions between neurons in the ventral horns of segment 14 in the adult Mosquitofish spinal cord (Figures 6-8); we found no gold beads on astrocyte or oligodendrocyte gap junctions (absence of labeling of glial gap junctions not shown). In one of two sagital section replicas of adult male Mosquitofish, we found 62 Cx35/36 immuno-positive gap junctions in segments containing the 8 th-16th ventral roots, with $>50$ of those gap junctions in neurons innervating the 14 th spinal segment ventral root (Figures 6-7).

Gap junctions at these glutamatergic mixed synapses are extraordinarily abundant in the 14th spinal segment (Figures 6-7), the main spinal segment that innervates the male sexually dimorphic genitalia, the gonopodium (Rosa-Molinar, 2005; Rivera-Rivera et al., 2010). Gap junctions are much less abundant in the 16th spinal segment and in the more rostral (1-7) spinal segments and in the more caudal (17-33) spinal segments of the adult (male and female) Mosquitofish spinal cord (data not shown). 

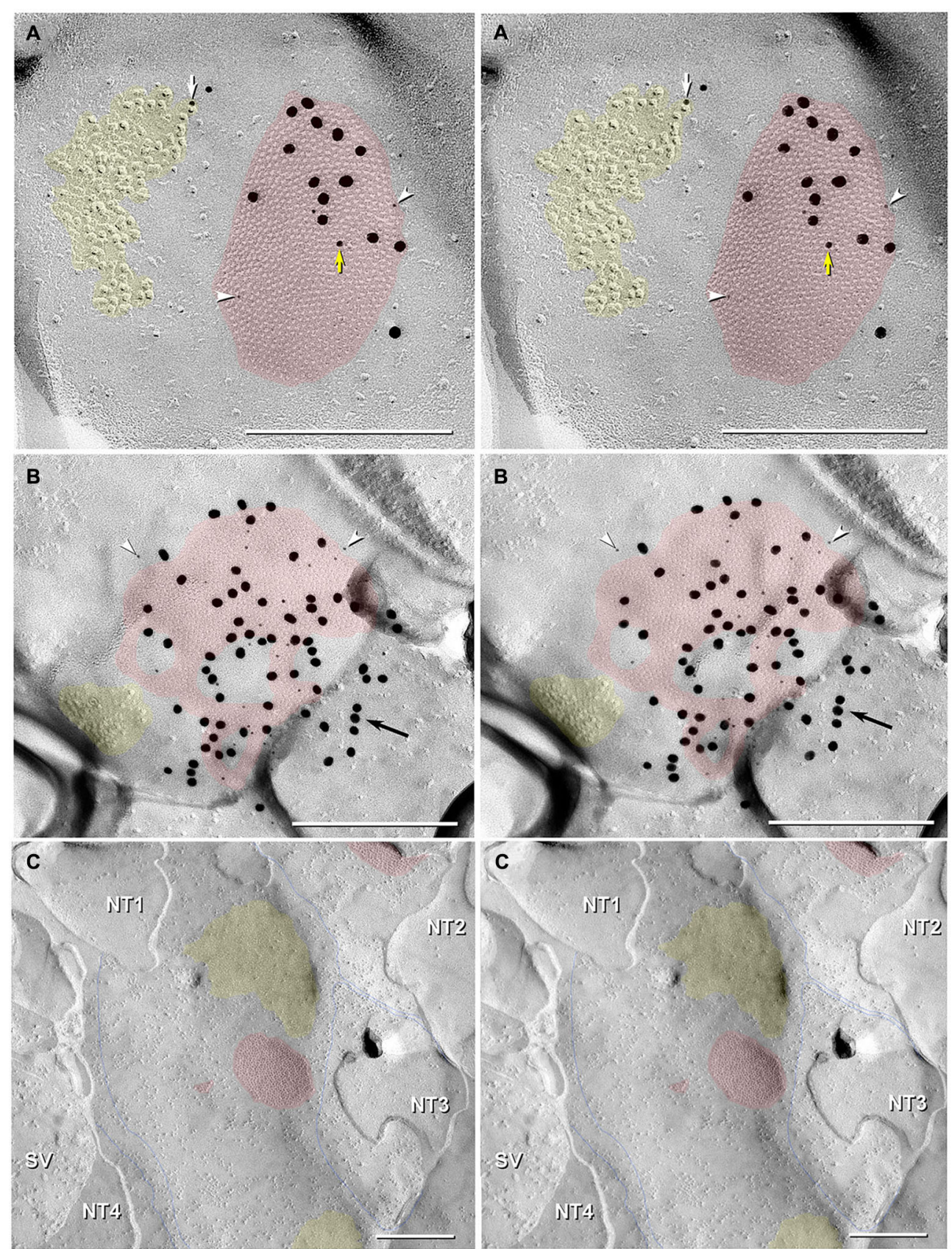

FIGURE 7 | Stereoscopic images of large plaque and reticular gap junctions in adult male Mosquitofish. (A) Large plaque gap junction (red overlay) on a neuronal postsynaptic E-face labeled with antibodies against mouse/human Cx36 (36-4600, 6-nm [white arrowheads] and much more electron-dense 18-nm gold beads) and for GluR2 AMPA receptors (12-nm gold beads, white arrow) (Yellow arrow indicates either a 12-nm gold bead as "noise" on the Cx36-labeled gap junction or an anomalously small "18-nm" gold bead for Cx36). (B) High-magnification stereoscopic image of E-face of a neuronal reticular gap junction (red overlay) labeled for Cx35 (P-Ser276) by 6-nm (white arrowheads) and 18-nm gold beads. The black arrow points to "cryptic" labeling of connexins in an extended portion of the gap junction beneath the cross-fractured neuronal cytoplasm. Immediately adjacent to the gap junction is a cluster of E-face
IMPs (yellow overlay, not labeled) similar to other immunogold-labeled glutamate receptor channels. (C) P-face image showing three of more than a dozen unlabeled postsynaptic hemiplaques (red overlays) in the same replica as Figures 4, 5B, where presynaptic hemiplaques are heavily labeled for Cx35 (P-Ser276). This absence of labeling in C is consistent with our demonstration in goldfish giant club ending/Mauthner cell mixed synapses that $\mathrm{C} \times 35$ is exclusively presynaptic and that $\mathrm{C} \times 34.7$ is exclusively postsynaptic (Rash et al., 2013). The postsynaptic P-face reveals distinct clusters of faintly resolvable pits (yellow overlays) where glutamate receptors had been removed during membrane splitting. Thin blue lines indicate probable margins of axon terminals impressed into the dendritic or somatic plasma membrane (NT1-NT4). SV = synaptic vesicles. Calibration bars are $0.25 \mu \mathrm{m}$ 
Gap junctions at mixed synapses between coupled MNs and CoPA INs usually are large ( $>400$ connexons) and are immunogold labeled by a variety of Cx35/36 antibodies. In ultrastructurally-identified mixed synapses, FRIL analysis reveals Cx35/36-labeled gap junctions adjacent to postsynaptic glutamate receptor E-face IMPs (see Pereda et al., 2003) in 43 out of 74 E-face images of mixed synapses (Figure 7, red vs. yellow overlays). These distinctive clusters of $10-\mathrm{nm}$ E-face particles are weakly labeled for NMDA (Figure 6) or for GluR2 (Figure 7A) glutamate receptors, thereby demonstrating that both NMDA and AMPA glutamate receptors are present and intermixed in the glutamate receptor clusters. These glutamatergic mixed synapses occur primarily on dendritic shafts (Figure 6A) and are only infrequently seen on dendritic spines (not shown). The antibodies used for identifying glutamate receptors were made to mammalian amino acid sequences that vary from the sequence in fish. As a result, the glutamate-receptor antibodies are only weakly cross-reactive with fish glutamate receptors, thus yielding a low but positive level of labeling. Pereda et al. (2003) previously documented the properties of these antibodies. In any case, the number, size, and clustering of the E-face IMPs in the glutamate receptor-labeled PSDs appear identical in mammals and fish.

In addition to typical "plaques", two large "reticular" gap junctions immunogold labeled for Cx35/36 were found in adult male Mosquitofish (Figure 7B); no reticular gap junctions have yet been found in female Mosquitofish. Reticular gap junctions are characterized by the presence of one or more oval areas that are devoid of connexon P-face IMPs / E-face pits (Kamasawa et al., 2006; Rash et al., 2007). Occasionally, the fracture plane stepped from the E-face to the P-face within the perimeter of a gap junction (Figure $\mathbf{8 A}$ from adult female Mosquitofish), thereby revealing the characteristic narrowing of the extracellular space (Figure 8A, blue overlay) at the contact area of gap junction coupling. Reverse stereoscopic imaging of Cx35/36 immunogold-labeled neuronal gap junctions (Figure 8; right pair of each triplet) facilitates discrimination of $6-\mathrm{nm}$ gold beads from the equally electron-opaque 6-9 $\mathrm{nm}$ platinum-shadowed IMPs (Figure 8A).

Finally, gold beads specifically labeling Cx35 but not Cx34.7 (antibody P-Ser276; Rash et al., 2013) are restricted to presynaptic hemiplaques of neuronal gap junctions (Figures 6, 7B) and do not label nearby postsynaptic hemiplaques in the same replica (Figure 7C). We previously showed the following: two anti-Cx36 antibodies cross-react with teleost $\mathrm{Cx} 35$ and $\mathrm{Cx} 34.7$ (Mouse antiConnexin 36 [39-4200; Figure 8A] and Ab298 [not used here]); two cross react with $\mathrm{Cx} 35$ but not $\mathrm{Cx} 34.7$ (37-4600 [Figure 8A] and Invitrogen 51-6300; not used here); two cross-react with Cx34.7 but not Cx35 (Cx34.7 IL and Cx34.7 CT; not used here);
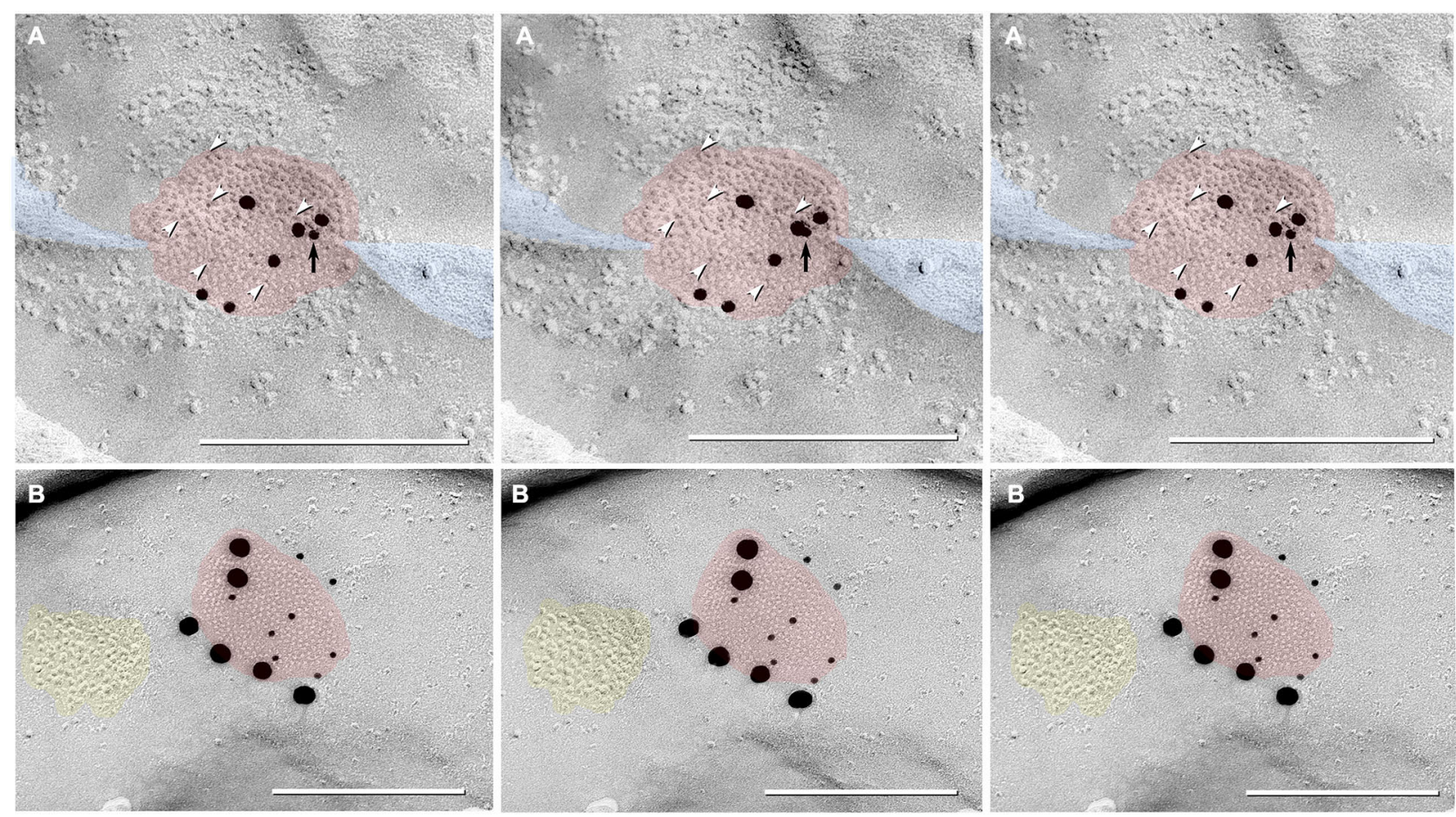

FIGURE 8 | Triplet images showing stereoscopic (left two of three images) and reverse stereoscopic images (right two images) of medium-size gap junction in adult female Mosquitofish. (A) Classical gap junction plaque (red overlays) labeled by 14 6-nm gold beads (six indicated by white arrowheads) and six 18-nm gold beads representing Cx36 (39-4200 and 37-4600). A single 12-nm gold nanoparticle (black arrow), ostensibly for anti-pannexin 1 (see Section Materials and Methods), represents non-specific labeling because it is on the non-biological side (upper surface) of the replica where labeling is not possible. Note the narrowing of the extracellular space (blue overlay) at the point of closest membrane approach within the gap junction. (B) E-face image of gap junction plaque (red overlay) labeled for Cx36 (36-4600) by eight 12-nm gold beads and six 30-nm gold beads. Because of greatly different amino acid sequences in fish vs. mammalian glutamate receptors (see text), the immediately adjacent cluster of glutamate receptor-like E-face IMPs (yellow overlay) does not exhibit labeling for GluR2 (6-nm and 18-nm gold beads, none present). Calibration bars are $0.25 \mu \mathrm{m}$. 
and one cross-reacts with Cx35 but has unknown cross-reactivity with Cx34.7 (36-4600 [Figures 7A, 8B]) (For antibody specificities, see Table $\mathrm{S} 1$ in Rash et al., 2013). In addition, phospho-specific P-Ser276 labels Cx35/Cx36 but not Cx34.7 (Figures 6, 7B,C).

Thus, the current data provide independent verification for Cx35/36 antibody specificity for labeling neuronal gap junctions but not glial gap junctions. In particular, current data confirm specificity of labeling for Cx35/Cx36 only in presynaptic hemiplaques but not in postsynaptic hemiplaques. Thus, as in goldfish, a second connexin homolog of Cx36 (likely Cx34.7; Rash et al., 2013) occurs in the otherwise unlabeled postsynaptic hemiplaques (Figure 7C). If so, these data from Mosquitofish imply that asymmetry of connexin distribution/heterotypic coupling may be widespread in teleost neuronal gap junctions.

\section{DISCUSSION}

The results support other studies that report gap junctions in the adult spinal cord as well as those that link gap junctions to motor behavior. Moreover, our results suggest mixed synapses have a role in the fast coital behavior of the adult male Mosquitofish, and, thus, extend prior studies (Gogan et al., 1974, 1977; Lewis, 1994; Laird, 1996; van der Want et al., 1998; Tresch and Kiehn, 2000; Kiehn and Tresch, 2002; Mentis et al., 2002; Arumugam et al., 2005; Park et al., 2011). Our findings show extensive dye-coupling between MNs and CoPA INs throughout the Mosquitofish spinal cord and permit the identification of two phenotypes of motor neurons, MN-1 and MN-2. Labeling reveals 44 neurons in the 14 th spinal segment that controls the fast coital movement of the male, and it also shows that the 14th spinal segment has the most extensive and elaborate dendritic arbor. The dyecoupling study shows that the number of mixed synapses falls off precipitously in more rostral (1-13) and in more caudal (17-33) spinal cord segments in both adult male and female Mosquitofish (data not shown). The latter three findings are consistent with a fast-responding role for mixed synapses in the adult male Mosquitofish spinal cord region linked to a malespecific coital behavior.

The labeling also shows abundant anti-Cx35/36 puncta surrounding the primary basal dendrite and the soma of Mosquitofish spinal neurons. The anti-Cx35/36-labeled puncta are widely distributed throughout the Mosquitofish spinal cord. The latter finding suggests that gap junctions may link a wide constellation of spinal neurons.

Because it is well accepted that anti-Cx35/36-labeled puncta are indicative of the occurrence of gap junctions between neurons, to confirm that dye-coupling between MNs and CoPA INs was mediated by Cx35-containing-gap junctions at mixed synapses, we employed FRIL and four Cx35/36 antibodies to define the ultrastructural details of the gap junctions. Results show 62 Cx35/36 immuno-positive gap junctions in segments $8-16$. In the 16 th spinal segment, the more rostral (1-7) spinal segments, and the more caudal (17-33) spinal segments of the adult male and female Mosquitofish, gap junctions are not as abundant as they are in the 14th spinal segment where $>50$ are shown.
Thus, in the main spinal segment ventral root (14th spinal segment) that innervates the male sexually dimorphic gonopodium, gap junctions at glutamatergic mixed synapses are extraordinarily abundant, reinforcing the suggestion that they have a role in fast behavior.

Although our findings contrast with those of Matsumoto et al. $(1988,1989)$ who found gap junction plaques only between MNs of the spinal nucleus of the bulbocavernosus (SNB) and the dorsolateral nucleus (DLN), they are in keeping with those of others. Coleman and Sengelaub (2002) reported dye-coupling between MNs and INs associated with the rodent SNB and the DLN, both of which are sexually dimorphic motor nuclei in the lumbosacral spinal cord involved in controlling genital reflexes. Although the Coleman and Sengelaub results should be replicated in response to the Bautista and Nagy (2014) questions regarding methodological issues and the observation that dye-coupling normally occurs between neurons of the same phenotype, the dye-coupling we observed between MNs and CoPA INs in the Mosquitofish spinal cord region associated with the sexually dimorphic ano-urogenital region supports and extends the Coleman and Sengelaub (2002) results. In addition, our results are in keeping with those of Rash and coworkers who first described gap junctions at neuronal mixed synapses throughout the spinal cord of adult rat (Rash et al., 1996, 1997, 1998, 2000, 2001; Rash and Yasumura, 1999).

In short, the independent use of "dye-coupling", wholemount immunofluorescence for gap junction channel protein connexin 35 (Cx35), and freeze-fracture replica labeling, show the abundance and persistence of gap junctions at glutamatergic mixed synapses in adult male and female Mosquitofish and provide a means for future studies to assign specific roles to spinal neurons in the generation/regulation of a sex-specific behavior.

The results establish a base for future studies to elucidate the idea that gap junctions have a major role in regulating neuronal aborization and morphology that directly affect spinal motor activity, particularly fast motor behavior, such as the male Mosquitofish "torque/thrust" maneuver. In addition, the gap junctions found at mixed synapses between MNs and CoPA INs suggest the CoPA IN may be a novel candidate neuron for future studies of an extended role for gap junctions in coordinating fast motor behavior.

\section{ACKNOWLEDGMENTS}

We thank John O'Brien for Cx35-specific antibody P-Ser276. We also thank N. L. Rivera-Rivera for characterizing the Cx35-specific antibody P-Ser276 and the mouse anti- Cx35/36 monoclonal antibodies, A. Pereda, M. Morita R. Diaz-De Leon, and E. SierraLopez for their helpful comments throughout the study, and J. Taylor for her editorial comments. This research was partially supported by grants to Eduardo Rosa-Molinar and Scott E. Fraser from NIH (NS 39405), NSF-1039620 (Eduardo RosaMolinar), NSF-0964114 (Eduardo Rosa-Molinar), Puerto Rico Science, Technology and Research Trust (Eduardo Rosa-Molinar Agreement 2013-000034); and NIH NS44395 (John E. Rash). Melanie Rodriguez-Alvarado was supported by a training grant from NIH (GM 007821). 


\section{REFERENCES}

Arumugam, H., Liu, X., Colombo, P. J., Corriveau, R. A., and Belousov, A. B. (2005). NMDA receptors regulate developmental gap junction uncoupling via CREB signaling. Nat. Neurosci. 8, 1720-1726. doi: 10.1038/nn1588

Bartos, M., Vida, I., Frotscher, M., Meyer, A., Monyer, H., Geiger, J. R., et al. (2002). Fast synaptic inhibition promotes synchronized gamma oscillations in hippocampal interneuron networks. Proc. Natl. Acad. Sci. U S A 99, 1322213227. doi: 10.1073/pnas.192233099

Bautista, W., and Nagy, J. I. (2014). Connexin36 in gap junctions forming electrical synapses between motoneurons in sexually dimorphic motor nuclei in spinal cord of rat and mouse. Eur. J. Neurosci. 39, 771-787. doi: 10.1111/ejn. 12439

Bennett, M. V. (1997). Gap junctions as electrical synapses. J. Neurocytol. 26, 349366. doi: 10.1023/A:1018560803261

Bennett, M. V. (2000). Electrical synapses, a personal perspective (or history). Brain Res. Brain Res. Rev. 32, 16-28. doi: 10.1016/s0165-0173(99)00065-x

Bennett, M. V., Aljure, E., Nakajima, Y., and Pappas, G. D. (1963). Electrotonic junctions between teleost spinal neurons: electrophysiology and ultrastructure. Science 141, 262-264. doi: 10.1126/science.141.3577.262

Bennett, M. V., and Zukin, R. S. (2004). Electrical coupling and neuronal synchronization in the Mammalian brain. Neuron 41, 495-511. doi: 10.1016/s08966273(04)00043-1

Bonnot, A., Whelan, P. J., Mentis, G. Z., and O'donovan, M. J. (2002). Spatiotemporal pattern of motoneuron activation in the rostral lumbar and the sacral segments during locomotor-like activity in the neonatal mouse spinal cord. $J$. Neurosci. 22:RC203. doi: 10.3410/f.1003953.42704

Chen, B., Liu, Q., Ge, Q., Xie, J., and Wang, Z. W. (2007). UNC-1 regulates gap junctions important to locomotion in C. elegans. Curr. Biol. 17, 1334-1339. doi: 10.1016/j.cub.2007.06.060

Chuang, C. F., Vanhoven, M. K., Fetter, R. D., Verselis, V. K., and Bargmann, C. I. (2007). An innexin-dependent cell network establishes left-right neuronal asymmetry in C. elegans. Cell 129, 787-799. doi: 10.1016/j.cell.2007. 02.052

Cohen-Cory, S. (2002). The developing synapse: construction and modulation of synaptic structures and circuits. Science 298, 770-776. doi: 10.1126/science. 1075510

Coleman, A. M., and Sengelaub, D. R. (2002). Patterns of dye coupling in lumbar motor nuclei of the rat. J. Comp. Neurol. 454, 34-41. doi: 10.1002/cne.10438

Connors, B. W., and Long, M. A. (2004). Electrical synapses in the mammalian brain. Annu. Rev. Neurosci. 27, 393-418. doi: 10.1146/annurev.neuro.26.041002. 131128

Costes, S. V., Daelemans, D., Cho, E. H., Dobbin, Z., Pavlakis, G., and Lockett, S. (2004). Automatic and quantitative measurement of protein-protein colocalization in live cells. Biophys. J. 86, 3993-4003. doi: 10.1529/biophysj.103.038422

DeFelipe, J., Conti, F., Van Eyck, S. L., and Manzoni, T. (1988). Demonstration of glutamate-positive axon terminals forming asymmetric synapses in cat neocortex. Brain Res. 455, 162-165. doi: 10.1016/0006-8993(88) 90127-8

Dermietzel, R. (1998). Gap junction wiring: a 'new' principle in cell-to-cell communication in the nervous system? Brain Res. Brain Res. Rev. 26, 176-183. doi: 10. 1016/s0165-0173(97)00031-3

Dermietzel, R., and Spray, D. C. (1993). Gap junctions in the brain: where, what type, how many and why? Trends Neurosci. 16, 186-192. doi: 10.1016/01662236(93)90151-b

Dinchuk, J. E., Johnson, T. J., and Rash, J. E. (1987). Postreplication labeling of Eleaflet molecules: membrane immunoglobulins localized in sectioned, labeled replicas examined by TEM and HVEM. J. Electron Microsc. Tech. 7, 1-16. doi: 10. 1002/jemt.1060070102

Domenici, P., and Blake, R. (1997). The kinematics and performance of fish faststart swimming. J. Exp. Biol. 200, 1165-1178.

Drapeau, P., Saint-Amant, L., Buss, R. R., Chong, M., Mcdearmid, J. R., and Brustein, E. (2002). Development of the locomotor network in zebrafish. Prog. Neurobiol. 68, 85-111. doi: 10.1016/s0301-0082(02)00075-8

Edwards, D. H., Heitler, W. J., and Krasne, F. B. (1999). Fifty years of a command neuron: the neurobiology of escape behavior in the crayfish. Trends Neurosci. 22, 153-161. doi: 10.1016/s0166-2236(98)01340-X

Fan, S. Y., Ke, Y. N., Zeng, Y. J., Wang, Y., Cheng, W. L., and Yang, J. R. (2005). [Effects and the mechanism of carvedilol on gap junctional intercellular communication in rat myocardium]. Zhonghua Xin Xue Guan Bing Za Zhi 33, 1141-1145.

Fujimoto, K. (1995). Freeze-fracture replica electron microscopy combined with SDS digestion for cytochemical labeling of integral membrane proteins. Application to the immunogold labeling of intercellular junctional complexes. J. Cell Sci. 108(Pt. 11), 3443-3449.

Fukuda, T., and Kosaka, T. (2000). Gap junctions linking the dendritic network of GABAergic interneurons in the hippocampus. J. Neurosci. 20, 1519-1528.

Fukuda, T., and Kosaka, T. (2003). Ultrastructural study of gap junctions between dendrites of parvalbumin-containing GABAergic neurons in various neocortical areas of the adult rat. Neuroscience 120, 5-20. doi: 10.1016/s03064522(03)00328-2

Fulton, B. P., Miledi, R., and Takahashi, T. (1980). Electrical synapses between motoneurons in the spinal cord of the newborn rat. Proc. R. Soc. Lond. B Biol. Sci. 208, 115-120. doi: 10.1098/rspb.1980.0045

Furshpan, E. J. (1964). "Electrical transmission" at an excitatory synapse in a vertebrate brain. Science 144, 878-880. doi: 10.1126/science.144.3620.878

Furshpan, E. J., and Potter, D. D. (1959). Transmission at the giant motor synapses of the crayfish. J. Physiol. 145, 289-325.

Galarreta, M., and Hestrin, S. (1999). A network of fast-spiking cells in the neocortex connected by electrical synapses. Nature 402, 72-75. doi: 10.1038/47029

Galarreta, M., and Hestrin, S. (2001a). Electrical synapses between GABA-releasing interneurons. Nat. Rev. Neurosci. 2, 425-433. doi: 10.1038/35077566

Galarreta, M., and Hestrin, S. (2001b). Spike transmission and synchrony detection in networks of GABAergic interneurons. Science 292, 2295-2299. doi: 10. 1126/science.1061395

Gibson, J. R., Beierlein, M., and Connors, B. W. (1999). Two networks of electrically coupled inhibitory neurons in neocortex. Nature 402, 75-79. doi: 10.1038/47035

Gogan, P., Gueritaud, J. P., Horcholle-Bossavit, G., and Tyc-Dumont, S. (1974). Electronic coupling between motoneurones in the abducens nucleus of the cat. Exp. Brain Res. 21, 139-154. doi: 10.1007/bf00234386

Gogan, P., Gueritaud, J. P., Horcholle-Bossavit, G., and Tyc-Dumont, S. (1977). Direct excitatory interactions between spinal motoneurones of the cat. J. Physiol. 272, 755-767.

Goodenough, D. A., and Paul, D. L. (2009). Gap junctions. Cold Spring Harb. Perspect. Biol. 1:a002576. doi: 10.1101/cshperspect.a002576

Hale, M. E. (2002). S- and C-start escape responses of the muskellunge (Esox masquinongy) require alternative neuromotor mechanisms. J. Exp. Biol. 205, 2005-2016.

Hale, M. E., Ritter, D. A., and Fetcho, J. R. (2001). A confocal study of spinal interneurons in living larval zebrafish. J. Comp. Neurol. 437, 1-16. doi: 10. 1002/cne.1266

Hamzei-Sichani, F., Davidson, K. G., Yasumura, T., Janssen, W. G., Wearne, S. L., Hof, P. R., et al. (2012). Mixed electrical-chemical synapses in adult rat hippocampus are primarily glutamatergic and coupled by connexin-36. Front. Neuroanat. 6:13. doi: 10.3389/fnana.2012.00013

Harper, D. G., and Blake, R. W. (1990). Fast-start performance of rainbow-trout Salmo-Gairdneri and Northern Pike Esox-Lucius. J. Exp. Biol. 150, 321-342.

Harper, D. G., and Blake, R. W. (1991). Prey capture and the fast-start performance of Northern Pike Esox-Lucius. J. Exp. Biol. 155, 175-192.

Harris, K. M., and Landis, D. M. (1986). Membrane structure at synaptic junctions in area CA1 of the rat hippocampus. Neuroscience 19, 857-872. doi: 10 . 1016/0306-4522(86)90304-0

Herberholz, J., Antonsen, B. L., and Edwards, D. H. (2002). A lateral excitatory network in the escape circuit of crayfish. J. Neurosci. 22, 9078-9085.

Hervé, J. C., Bourmeyster, N., and Sarrouilhe, D. (2004). Diversity in proteinprotein interactions of connexins: emerging roles. Biochim. Biophys. Acta 1662, 22-41. doi: 10.1016/j.bbamem.2003.10.022

Hoge, G. J., Davidson, K. G., Yasumura, T., Castillo, P. E., Rash, J. E., and Pereda, A. E. (2011). The extent and strength of electrical coupling between inferior olivary neurons is heterogeneous. J. Neurophysiol. 105, 1089-1101. doi: 10. 1152/jn.00789.2010

Johnston, I. I., Leeuwen, J., Davies, M., and Beddow, T. (1995). How fish power predation fast-starts. J. Exp. Biol. 198, 1851-1861.

Kalb, R. G. (1994). Regulation of motor neuron dendrite growth by NMDA receptor activation. Development 120, 3063-3071.

Kamasawa, N., Furman, C. S., Davidson, K. G., Sampson, J. A., Magnie, A. R., Gebhardt, B. R., et al. (2006). Abundance and ultrastructural diversity of 
neuronal gap junctions in the OFF and ON sublaminae of the inner plexiform layer of rat and mouse retina. Neuroscience 142, 1093-1117. doi: 10.1016/j. neuroscience.2006.08.020

Kandler, K., and Katz, L. C. (1995). Neuronal coupling and uncoupling in the developing nervous system. Curr. Opin. Neurobiol. 5, 98-105. doi: 10.1016/09594388(95)80093-x

Kiehn, O., and Tresch, M. C. (2002). Gap junctions and motor behavior. Trends Neurosci. 25, 108-115. doi: 10.1016/s0166-2236(02)02038-6

Laird, D. W. (1996). The life cycle of a connexin: gap junction formation, removal and degradation. J. Bioenerg. Biomembr. 28, 311-318. doi: 10.1007/bf02 110107

Lewis, D. I. (1994). Dye-coupling between vagal motoneurones within the compact region of the adult rat nucleus ambiguus, in-vitro. J. Auton. Nerv. Syst. 47, 53-58. doi: 10.1016/0165-1838(94)90065-5

Lewis, K. E., and Eisen, J. S. (2003). From cells to circuits: development of the zebrafish spinal cord. Prog. Neurobiol. 69, 419-449. doi: 10.1016/s03010082(03)00052-2

Li, H., Chuang, A. Z., and O’brien, J. (2009). Photoreceptor coupling is controlled by connexin 35 phosphorylation in zebrafish retina. J. Neurosci. 29, 15178 15186. doi: 10.1523/JNEUROSCI.3517-09.2009

Lynn, B. D., Li, X., and Nagy, J. I. (2012). Under construction: building the macromolecular superstructure and signaling components of an electrical synapse. $J$. Membr. Biol. 245, 303-317. doi: 10.1007/s00232-012-9451-5

Marin-Burgin, A., Eisenhart, F. J., Baca, S. M., Kristan, W. B. Jr., and French, K. A. (2005). Sequential development of electrical and chemical synaptic connections generates a specific behavioral circuit in the leech. J. Neurosci. 25, 2478-2489. doi: 10.1523/jneurosci.4787-04.2005

Marin-Burgin, A., Eisenhart, F. J., Kristan, W. B. Jr., and French, K. A. (2006). Embryonic electrical connections appear to pre-figure a behavioral circuit in the leech CNS. J. Comp. Physiol. A Neuroethol. Sens. Neural. Behav. Physiol. 192, 123-133. doi: 10.1007/s00359-005-0055-8

Marin-Burgin, A., Kristan, W. B. Jr., and French, K. A. (2008). From synapses to behavior: development of a sensory-motor circuit in the leech. Dev. Neurobiol. 68, 779-787. doi: 10.1002/dneu.20551

Matsumoto, A., Arnold, A. P., and Micevych, P. E. (1989). Gap junctions between lateral spinal motoneurons in the rat. Brain Res. 495, 362-366. doi: 10. 1016/0006-8993(89)90229-1

Matsumoto, A., Arnold, A. P., Zampighi, G. A., and Micevych, P. E. (1988). Androgenic regulation of gap junctions between motoneurons in the rat spinal cord. J. Neurosci. 8, 4177-4183.

McAllister, A. K. (2007). Dynamic aspects of CNS synapse formation. Annu. Rev. Neurosci. 30, 425-450. doi: 10.1146/annurev.neuro.29.051605.112830

Mentis, G. Z., Diaz, E., Moran, L. B., and Navarrete, R. (2002). Increased incidence of gap junctional coupling between spinal motoneurones following transient blockade of NMDA receptors in neonatal rats. J. Physiol. 544, 757-764. doi: 10. 1113/jphysiol.2002.028159

Montoro, R. J., and Yuste, R. (2004). Gap junctions in developing neocortex: a review. Brain Res. Brain Res. Rev. 47, 216-226. doi: 10.1016/j.brainresrev.2004. 06.009

Muller, J. F., Mascagni, F., and Mcdonald, A. J. (2005). Coupled networks of parvalbumin-immunoreactive interneurons in the rat basolateral amygdala. J. Neurosci. 25, 7366-7376. doi: 10.1523/jneurosci.0899-05.2005

Muller, J. F., Mascagni, F., and Mcdonald, A. J. (2006). Pyramidal cells of the rat basolateral amygdala: synaptology and innervation by parvalbuminimmunoreactive interneurons. J. Comp. Neurol. 494, 635-650. doi: 10.1002/cne. 20832

Norman, K. R., and Maricq, A. V. (2007). Innexin function: minding the gap junction. Curr. Biol. 17, R812-R814. doi: 10.1016/j.cub.2007.07.043

Park, W. M., Wang, Y., Park, S., Denisova, J. V., Fontes, J. D., and Belousov, A. B. (2011). Interplay of chemical neurotransmitters regulates developmental increase in electrical synapses. J. Neurosci. 31, 5909-5920. doi: 10. 1523/JNEUROSCI.6787-10.2011

Peinado, A., Yuste, R., and Katz, L. C. (1993a). Extensive dye coupling between rat neocortical neurons during the period of circuit formation. Neuron 10, 103-114. doi: 10.1016/0896-6273(93)90246-n

Peinado, A., Yuste, R., and Katz, L. C. (1993b). Gap junctional communication and the development of local circuits in neocortex. Cereb. Cortex 3, 488-498. doi: 10. $1093 /$ cercor/3.5.488
Pereda, A., O’brien, J., Nagy, J. I., Bukauskas, F., Davidson, K. G., Kamasawa, N., et al. (2003). Connexin 35 mediates electrical transmission at mixed synapses on Mauthner cells. J. Neurosci. 23, 7489-7503.

Pereda, A. E., Curti, S., Hoge, G., Cachope, R., Flores, C. E., and Rash, J. E. (2013). Gap junction-mediated electrical transmission: regulatory mechanisms and plasticity. Biochim. Biophys. Acta 1828, 134-146. doi: 10.1016/j.bbamem. 2012.05.026

Personius, K., Chang, Q., Bittman, K., Panzer, J., and Balice-Gordon, R. (2001). Gap junctional communication among motor and other neurons shapes patterns of neural activity and synaptic connectivity during development. Cell Commun. Adhes. 8, 329-333. doi: 10.3109/15419060109080748

Peters, A., Palay, S. L., and Webster, H. D. (1991). The Fine Structure of the Nervous System. Neurons and Their Supporting Cells. New York: Oxford University Press.

Phelan, P. (2005). Innexins: members of an evolutionarily conserved family of gap-junction proteins. Biochim. Biophys. Acta 1711, 225-245. doi: 10.1016/j. bbamem.2004.10.004

Rash, J. E., Curti, S., Vanderpool, K. G., Kamasawa, N., Nannapaneni, S., Palacios-Prado, N., et al. (2013). Molecular and functional asymmetry at a vertebrate electrical synapse. Neuron 79, 957-969. doi: 10.1016/j.neuron.2013. 06.037

Rash, J. E., Davidson, K. G., Kamasawa, N., Yasumura, T., Kamasawa, M., Zhang, C., et al. (2005). Ultrastructural localization of connexins (Cx36, Cx43, Cx45), glutamate receptors and aquaporin-4 in rodent olfactory mucosa, olfactory nerve and olfactory bulb. J. Neurocytol. 34, 307-341. doi: 10.1007/s11068-0058360-2

Rash, J. E., Dillman, R. K., Bilhartz, B. L., Duffy, H. S., Whalen, L. R., and Yasumura, T. (1996). Mixed synapses discovered and mapped throughout mammalian spinal cord. Proc. Natl. Acad. Sci. U S A 93, 4235-4239. doi: 10.1073/pnas.93. 9.4235

Rash, J. E., Duffy, H. S., Dudek, F. E., Bilhartz, B. L., Whalen, L. R., and Yasumura, T. (1997). Grid-mapped freeze-fracture analysis of gap junctions in gray and white matter of adult rat central nervous system, with evidence for a "panglial syncytium" that is not coupled to neurons. J. Comp. Neurol. 388, 265-292. doi: 10.1002/(sici)1096-9861(19971117)388:2<265::aid-cne6>3.0.co;2-\#

Rash, J. E., Olson, C. O., Davidson, K. G., Yasumura, T., Kamasawa, N., and Nagy, J. I. (2007). Identification of connexin36 in gap junctions between neurons in rodent locus coeruleus. Neuroscience 147, 938-956. doi: 10.1016/j.neuroscience. 2007.04.061

Rash, J. E., Pereda, A., Kamasawa, N., Furman, C. S., Yasumura, T., Davidson, K. G., et al. (2004). High-resolution proteomic mapping in the vertebrate central nervous system: close proximity of connexin35 to NMDA glutamate receptor clusters and co-localization of connexin 36 with immunoreactivity for zonula occludens protein-1 (ZO-1). J. Neurocytol. 33, 131-151. doi: 10.1023/B:NEUR. 0000029653.34094.0b

Rash, J. E., Staines, W. A., Yasumura, T., Patel, D., Furman, C. S., Stelmack, G. L., et al. (2000). Immunogold evidence that neuronal gap junctions in adult rat brain and spinal cord contain connexin-36 but not connexin-32 or connexin43. Proc. Natl. Acad. Sci. U S A 97, 7573-7578. doi: 10.1073/pnas.97.13.7573

Rash, J. E., Yasumura, T., and Dudek, F. E. (1998). Ultrastructure, histological distribution and freeze-fracture immunocytochemistry of gap junctions in rat brain and spinal cord. Cell Biol. Int. 22, 731-749. doi: 10.1006/cbir.1998.0392

Rash, J. E., Yasumura, T., Davidson, K. G., Furman, C. S., Dudek, F. E., and Nagy, J. I. (2001). Identification of cells expressing Cx43, Cx30, Cx26, Cx32 and Cx36 in gap junctions of rat brain and spinal cord. Cell Commun. Adhes. 8, 315-320. doi: 10.3109/15419060109080745

Rash, J. E., and Yasumura, T. (1999). Direct immunogold labeling of connexins and aquaporin-4 in freeze-fracture replicas of liver, brain and spinal cord: factors limiting quantitative analysis. Cell Tissue Res. 296, 307-321. doi: 10. 1007/s004410051291

Rivera-Rivera, N. L., Martinez-Rivera, N., Torres-Vazquez, I., Serrano-Velez, J. L., Lauder, G. V., and Rosa-Molinar, E. (2010). A male poecillid's sexually dimorphic body plan, behavior and nervous system. Integr. Comp. Biol. 50, 1081-1090. doi: $10.1093 / \mathrm{icb} / \mathrm{icq} 147$

Robertson, J. D., Bodenheimer, T. S., and Stage, D. E. (1963). The ultrastructure of mauthner cell synapses and nodes in goldfish brains. J. Cell Biol. 19, 159-199. doi: $10.1083 /$ jcb.19.1.159

Rosa-Molinar, E. (2005). "Edwin S. Goodrich's theory of transposition revisited: the shift to a sexually dimorphic axial formulae and nervous system in a 
poeciliine fish," in First International Symposium on Livebearing Fishes, eds M. C. Uribe and H. J. Grier (Homestead, Florida: New Life Publications, Inc.), $1-12$.

Rosa-Molinar, E., Fritzsch, B., and Hendricks, S. E. (1996). Organizationalactivational concept revisited: sexual differentiation in an atherinomorph teleost. Horm. Behav. 30, 563-575. doi: 10.1006/hbeh.1996.0059

Rosa-Molinar, E., Hendricks, S. E., Rodriguez-Sierra, J. F., and Fritzsch, B. (1994). Development of the anal fin appendicular support in the western mosquitofish, Gambusia affinis affinis (Baird and Girard, 1854): a reinvestigation and reinterpretation. Acta Anat. (Basel) 151, 20-35. doi: 10.1159/000147639

Rosa-Molinar, E., Proskocil, B. J., Hendricks, S. E., and Fritzsch, B. (1998). A mechanism for anterior transposition of the anal fin and its appendicular support in the western mosquitofish, gambusia affinis affinis. Acta Anat. (Basel) 163, 75-91. doi: 10.1159/000046487

Rosario-Ortiz, L., Rivera-Pabon, S., Torres-Vazquez, I., Ortiz-Perez, E. G., DiazPerez, L., Pesquera-Diaz, L., et al. (2008). The Western Mosquitofish (Gambusia affinis affinis): a new laboratory animal resource for the study of sexual dimorphism in neural circuits. Lab. Anim. (NY) 37, 263-269. doi: 10.1038/laban 0608-263

Saint-Amant, L., and Drapeau, P. (2000). Motoneuron activity patterns related to the earliest behavior of the zebrafish embryo. J. Neurosci. 20, 3964-3972.

Saint-Amant, L., and Drapeau, P. (2001). Synchronization of an embryonic network of identified spinal interneurons solely by electrical coupling. Neuron 31 , 1035-1046. doi: 10.1016/s0896-6273(01)00416-0

Scheiffele, P. (2003). Cell-cell signaling during synapse formation in the CNS. Annu. Rev. Neurosci. 26, 485-508. doi: 10.1146/annurev.neuro.26.043002.094940

Söhl, G., Maxeiner, S., and Willecke, K. (2005). Expression and functions of neuronal gap junctions. Nat. Rev. Neurosci. 6, 191-200. doi: 10.1038/nrn 1627

Sotelo, C., and Korn, H. (1978). Morphological correlates of electrical and other interactions through low-resistance pathways between neurons of the vertebrate central nervous system. Int. Rev. Cytol. 55, 67-107. doi: 10.1016/s00747696(08)61887-2

Spierts, I. L., and Leeuwen, J. L. (1999). Kinematics and muscle dynamics of C- and S-starts of carp (Cyprinus carpio L.). J. Exp. Biol. 202, 393-406.

Sugimoto, M., Sasaki, S., Gotoh, Y., Nakamura, Y., Aoyagi, Y., Kawahara, T., et al. (2013). Genetic variants related to gap junctions and hormone secretion influence conception rates in cows. Proc. Natl. Acad. Sci. U S A 110, 1949519500. doi: $10.1073 /$ pnas. 1309307110

Szabo, T. M., and Zoran, M. J. (2007). Transient electrical coupling regulates formation of neuronal networks. Brain Res. 1129, 63-71. doi: 10.1016/j.brainres. 2006.09.112

Szabo, T. M., Faber, D. S., and Zoran, M. J. (2004). Transient electrical coupling delays the onset of chemical neurotransmission at developing synapses. J. Neurosci. 24, 112-120. doi: 10.1523/jneurosci.4336-03.2004

Todd, K. L., Kristan, W. B. Jr., and French, K. A. (2010). Gap junction expression is required for normal chemical synapse formation. J. Neurosci. 30, 15277-15285. doi: 10.1523/jneurosci.2331-10.2010

Tresch, M. C., and Kiehn, O. (2000). Motor coordination without action potentials in the mammalian spinal cord. Nat. Neurosci. 3, 593-599. doi: 10.1038/75768

van der Want, J. J., Gramsbergen, A., Ijkema-Paassen, J., De Weerd, H., and Liem, R. S. (1998). Dendro-dendritic connections between motoneurons in the rat spinal cord: an electron microscopic investigation. Brain Res. 779, 342-345. doi: 10.1016/s0006-8993(97)01238-9

Vaney, D. I. (1991). Many diverse types of retinal neurons show tracer coupling when injected with biocytin or neurobiotin. Neurosci. Lett. 125, 187-190. doi: 10.1016/0304-3940(91)90024-n

Vega, I. E., Cui, L., Propst, J. A., Hutton, M. L., Lee, G., and Yen, S. H. (2005). Increase in tau tyrosine phosphorylation correlates with the formation of tau aggregates. Brain Res. Mol. Brain Res. 138, 135-144. doi: 10.1016/j.molbrainres. 2005.04.015

Vega, I. E., Traverso, E. E., Ferrer-Acosta, Y., Matos, E., Colon, M., Gonzalez, J., et al. (2008). A novel calcium-binding protein is associated with tau proteins in tauopathy. J. Neurochem. 106, 96-106. doi: 10.1111/j.1471-4159.2008. 05339.x

Vervaeke, K., Lorincz, A., Gleeson, P., Farinella, M., Nusser, Z., and Silver, R. A. (2010). Rapid desynchronization of an electrically coupled interneuron network with sparse excitatory synaptic input. Neuron 67, 435-451. doi: 10.1016/j. neuron.2010.06.028

Waites, C. L., Craig, A. M., and Garner, C. C. (2005). Mechanisms of vertebrate synaptogenesis. Annu. Rev. Neurosci. 28, 251-274. doi: 10.1146/annurev.neuro. 27.070203.144336

Walton, K. D., and Navarrete, R. (1991). Postnatal changes in motoneurone electrotonic coupling studied in the in vitro rat lumbar spinal cord. J. Physiol. $433,283-305$.

Webb, P. W. (1976). The effect of size on the fast-start performance of rainbow trout Salmo cairdneri and a consideration of piscivorous predator-prey interactions. J. Exp. Biol. 65, 157-177.

Weihs, D. (1973). The mechanism of rapid starting of slender fish. Biorheology 10, 343-350.

Whelan, P. J. (2010). Shining light into the black box of spinal locomotor networks. Philos. Trans. R Soc. Lond. B Biol. Sci. 365, 2383-2395. doi: 10.1098/rstb.2009. 0322

Wolszon, L. (1995). Cell-cell interactions define the innervation patterns of central leech neurons during development. J. Neurobiol. 27, 335-352. doi: 10.1002/neu. 480270307

Conflict of Interest Statement: The authors declare that the research was conducted in the absence of any commercial or financial relationships that could be construed as a potential conflict of interest.

Received: 16 February 2014; accepted: 28 May 2014; published online: 26 June 2014. Citation: Serrano-Velez JL, Rodriguez-Alvarado M, Torres-Vazquez II, Fraser SE, Yasumura T, Vanderpool KG, Rash JE and Rosa-Molinar E (2014) Abundance of gap junctions at glutamatergic mixed synapses in adult Mosquitofish spinal cord neurons. Front. Neural Circuits 8:66. doi: 10.3389/fncir.2014.00066 This article was submitted to the journal Frontiers in Neural Circuits.

Copyright (c) 2014 Serrano-Velez, Rodriguez-Alvarado, Torres-Vazquez, Fraser, Yasumura, Vanderpool, Rash and Rosa-Molinar. This is an open-access article distributed under the terms of the Creative Commons Attribution License (CC BY). The use, distribution or reproduction in other forums is permitted, provided the original author(s) or licensor are credited and that the original publication in this journal is cited, in accordance with accepted academic practice. No use, distribution or reproduction is permitted which does not comply with these terms. 